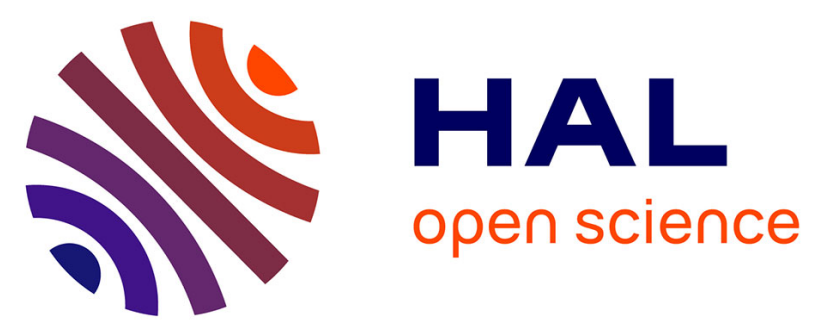

\title{
Challenging Top Level Aircraft Requirements based on operations analysis and data-driven models, application to take-off performance design requirements
}

Nicolas Peteilh, Thierry Klein, Thierry Druot, Nathalie Bartoli, Rhea P Liem

\section{- To cite this version:}

Nicolas Peteilh, Thierry Klein, Thierry Druot, Nathalie Bartoli, Rhea P Liem. Challenging Top Level Aircraft Requirements based on operations analysis and data-driven models, application to take-off performance design requirements. AIAA AVIATION 2020 FORUM, American Institute of Aeronautics and Astronautics, Jun 2020, Reno, NV, United States. 10.2514/6.2020-3171 . hal-02866381

\section{HAL Id: hal-02866381 \\ https://hal-enac.archives-ouvertes.fr/hal-02866381}

Submitted on 12 Jun 2020

HAL is a multi-disciplinary open access archive for the deposit and dissemination of scientific research documents, whether they are published or not. The documents may come from teaching and research institutions in France or abroad, or from public or private research centers.
L'archive ouverte pluridisciplinaire HAL, est destinée au dépôt et à la diffusion de documents scientifiques de niveau recherche, publiés ou non, émanant des établissements d'enseignement et de recherche français ou étrangers, des laboratoires publics ou privés. 


\title{
Challenging Top Level Aircraft Requirements based on operations analysis and data-driven models, application to take-off performance design requirements
}

\author{
Nicolas Peteilh *, Thierry Klein ${ }^{\dagger}$ and Thierry Druot ${ }^{\ddagger}$ \\ Ecole Nationale de l'Aviation Civile, ENAC Lab, Université de Toulouse, France \\ Nathalie Bartoli ${ }^{\S}$ \\ ONERA/DTIS, Université de Toulouse, France \\ Rhea P. Liem II \\ Hong Kong University of Science and Technology (HKUST), Hong Kong SAR
}

In an aircraft design process, Top Level Aircraft Requirements (TLAR) summarize the expected performance of future aircraft. They include regulatory, technological, industrial, economic, environment and operational performance requirements. The market analysis, forecasts, and strategic issues are key information in setting these requirements. Some of them can be challenging and may result in overdesigned aircraft, with a direct consequence in increased fuel consumption and cost. As such, TLARs often need to be renegotiated based on sensitivity studies. In designing an aircraft, some of these TLARs can be modeled as constraints in the optimization problem, such as the take-off requirement consideration. The objective of this paper is to propose a new method to incorporate more information in the renegotiation process by turning some TLARs from constraints to design variables. We analyze the impacts of these take-off performance requirements on the aircraft operational costs by performing sensitivity analyses. In particular, we investigate the tradeoff caused by the relaxed requirements: the reduction in overall cost associated with the redesigning of aircraft (i.e., no longer overdesigned) and the income loss and potential additional cost due to the reduced maximum payload that has to be sacrificed under some circumstances (e.g., wet runways). To perform this study, we rely on a calibrated conceptual aircraft design library and operational data analysis. The take-off field length is found to be the parameter having the greatest impact on operation costs. A new data driven objective function taking into account cost penalty resulting from reduced performance is used. The results show that another optimum exists and that combining airplane design and operations can provide new perspectives. Global sensitivity analysis and new generalized index appear promising to bring new information in the design process.

\section{Nomenclature}

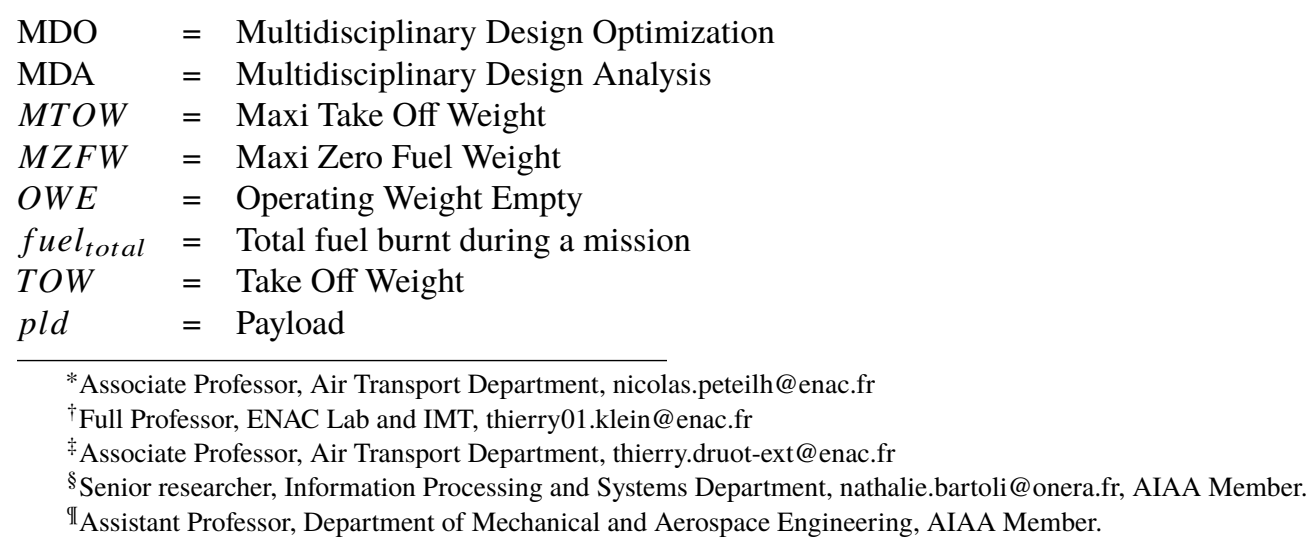




\section{Introduction}

Defining the key mission requirements is a key initial step in any new aircraft design projects. It is therefore important to define the relevant Top Level Aircraft Requirements (TLARs) in order to trigger and drive the design activity. A chapter of the TLARs which has a great impact on the future product concerns the operational performances. Up to now, the perception of the market needs and so the TLARs are built from market analysis, forecasts, and strategic issues. In most of the cases, some airlines are specifically targeted which may lead to very precise requirements in term of operational performances and weather conditions based on the geographical locations. The desire to shape the most attractive product versus competition frequently raises the tendency to determine the operational requirements on the basis of a worst case approach about the weather conditions regardless of how many times in the year the aircraft will be facing effectively such conditions. For instance, a targeted airline may want the airplane to be able to take off from a given airport located at an altitude of 6000ft, at Maximum Design Take Off Weight in ISA+34 with fifteen knots of tail wind and a wet runway. If an aircraft would finally be designed with such very challenging requirements, there is a high chance that it would be oversized for its common usage with a direct consequence in increased fuel consumption and finally cost. That is the reason why TLARs are most often readjusted through a negotiation process based on sensitivity studies.

In an airplane design process, the TLARs are modeled and included in different parts of the multidisciplinary process. Some of the TLARs form a set of constraints that the final aircraft design must comply with. The operational performance ones such as the approach speed, time to climb, vertical speed during climb or cruise are some examples. Among them, take-off requirements often appear as limiting constraints for the design of a new airplane. These requirements are stated as a combination of mass, take-off distance, meteorological conditions such as temperature, atmospheric pressure and wind at the time of take off, and other airport environment conditions like the runway condition (dry or wet) or the existence of potential obstacles close to the expected flight paths. Taking off from a high-altitude airport on a hot day and a wet runway, for instance, is typically considered as a challenging operational situation from a maximum payload and range points of view. Airlines expect hardly any or no limitations up to certain conditions.

The consequence of meeting these severe requirements results in heavier airplane and higher fuel consumption for all flights although they correspond to exceptional operational conditions. This triggers the following questions.

1) Considering the design process, how much do the take-off requirements impact operation costs?

2) Considering past and present operations, how often do these extreme take-off conditions occur?

3) Considering redesign with less severe take-off requirements:

- How much would airlines save on everyday operations?

- How much would the reduced performances cost to airlines?

4) What would be the best TLARs to minimize airlines operational costs and the best way to define them?

The first part of this paper presents the design process tool used for the analysis and the operational data used as a basis for challenging TLARs. The second part describes the method that was put in place to challenge the TLARs related to take-off performances. The third one presents and discusses the results obtained.

\section{Operational data and airplane design tools}

The study presented in this paper relies on one hand on an airplane design tool and on the other hand on a database.

\section{A. Airplane design tool}

The airplane design tool is based on the MARILib library [1]. MARILib contains a set of models dedicated to airplane conceptual design, enabling different formulations of multidisciplinary design analysis and optimization (MDAO). MARILib can therefore be used for MDO algorithms benchmark. It offers generic low fidelity models that enables to size modern conventional and unconventional aircraft based on a reduced number of top level aircraft requirements (TLARs), including number of passengers, range, cruise mach, but also constraints such as time-to-climb, climb and cruise ceilings, approach speed, one-engine-inoperative flight path and take-off requirements. In this study, we focus on conventional turbofan aircraft and more particularly the A340-300. This choice is made based on the available database, presented in Section III.B. In the rest of this paper, the A340-300 will be designated as the reference aircraft. Nevertheless, the method described here is more general and could be used for any other reference aircraft.

The generic low fidelity models (also referred to as functions) have therefore be tuned in order to match the A340-300. The general sequence of calculations made by MARILib to size an aircraft was presented in [1]. This process includes 
feedback loops, which are characteristics in any multidisciplinary analysis (MDA) and optimization (MDO) processes. We have defined a new specific process used for tuning that removes all the couplings of the MDA and runs through all the functions. This process is therefore purely sequential.

To run the different models, it is necessary to initialize some parameters based on the TLARs. The tuning process is then split in two phases:

1) the initialization phase runs all the functions needed to initialize some parameters,

2) the sizing phase that sequentially runs all the functions of the original MDA process.

As a result, and even if all the couplings have been removed, few parameters (such as the wing area) are calculated twice (once in the initialization phase and a second time in the sizing phase). The method used to tune the initialization functions is identical to the one used for the sizing functions. We focus now on the tuning process for the sizing phase. Each of the $N$ parameters $x_{i}, i \in\{1 . . N\}$ are sequentially computed i.e. $x_{i}$ is calculated before $x_{j}$ as soon as $i<j$. We also note the set of parameters $X_{i-1}=\left\{x_{j} \mid j \in\{1 . . i-1\}\right.$. If $i=1$, then, $X_{i-1}=\emptyset$. The process is represented in Equation (1) by a sequence of functions $f_{i}, \forall i \in\{1 . . N\}$ :

$$
\forall i \in\{1 . . N\}, x_{i}=f_{i}\left(T L A R s, X_{i-1}\right)
$$

Note that each function $f_{i}$ is therefore associated to one unique $x_{i}$.

For the reference aircraft, we use publicly available data, expert evaluation or internally available data at the ENAC to find the reference value corresponding to each $x_{i}$ and noted $x_{i}{ }^{\text {ref }}$. The basic principle for tuning aims at calculating, for each $x_{i}$ one tuning coefficient, chosen by expert judgement, between the two following types of coefficient:

- $\alpha_{i}$ which is a multiplying coefficient:

$$
x_{i}=\alpha_{i} f_{i}\left(T L A R s, X_{i-1}\right)
$$

- $\beta_{i}$ which is an additive coefficient:

$$
x_{i}=f_{i}\left(T L A R s, X_{i-1}\right)+\beta_{i}
$$

The tuning (or calibration) process therefore consists, for each $i$ and before considering the next parameter $x_{i+1}$, to calculate either $\alpha_{i}$ or $\beta_{i}$ thanks to Equation (2).

$$
\begin{aligned}
\alpha_{i} & =x_{i}{ }^{\text {ref }} / f_{i}\left(\text { TLARs }, X_{i-1}\right) \\
\beta_{i} & =x_{i}{ }^{\text {ref }}-f_{i}\left(\text { TLARs }, X_{i-1}\right)
\end{aligned}
$$

This method ensures that the coefficients $\alpha_{i}$ and $\beta_{i}$ specifically address the tuning of $f_{i}$. The general sequence used for the tuning is shown as follows:

1) Geometry: Figure 1 shows the three view geometry of the calibrated aircraft,

2) Mass,

3) Propulsion: Figure 2 presents the relative errors made on the thrust, the specific fuel consumption and the fuel flow for different values of mach and altitude,

4) Aerodynamics: a comparison of the polar curve (lift-over-drag versus lift coefficient) is shown in Figure 3 , between the "real" aircraft and the calibrated MARILib one,

5) Mission: Figure 4 shows a flight profile example, where the airplane changes flight level based on best specific air range and when performance ceilings allows it,

6) Performances: for example, Figure 5 shows operational climb and cruise ceilings.

$$
\left\{\begin{array}{l}
M Z F W=O W E(M T O W, M L W, M Z F W)+\text { payload }_{\text {max }} \\
M L W=\min (M T O W, 1.07 \times M Z F W) \\
M T O W=O W E(M T O W, M L W, M Z F W)+\text { payload } \text { fuel }_{\text {total }}(O W E)
\end{array}\right.
$$

Once all the tuning coefficients are calculated, the next step is to ensure that the MDA process (including the mass-mission loop) actually produces an airplane equivalent to the reference airplane. As this coupling aims to solve the set of non linear equations presented in Equation (3), we ensure neutral tuning coefficients to both $M Z F W, M L W$ and $M T O W$. Indeed, these expressions represent the definition of these masses. A non neutral coefficient would mean these definitions should be reviewed. As a consequence, we must set $\alpha_{M T O W}=1, \alpha_{M Z F W}=1$, and $\alpha_{M L W}=1$.

Finally, the last challenge was to ensure that the MDO process used to solve the constrained optimization problem converges towards the reference aircraft. As the MDA process already produces the reference aircraft, the constraints 


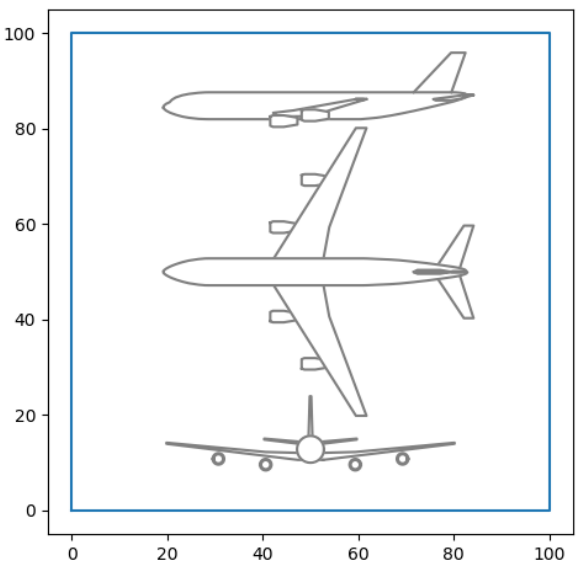

Fig. 1 Three view drawing of the calibrated aircraft.

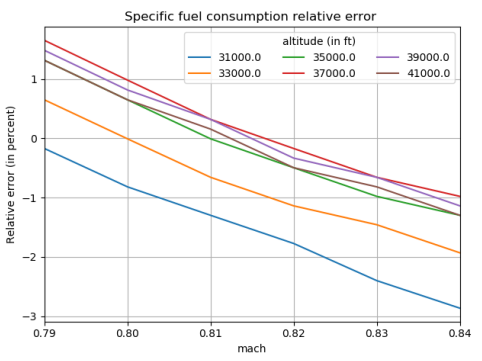

(a) $S F C$ relative error

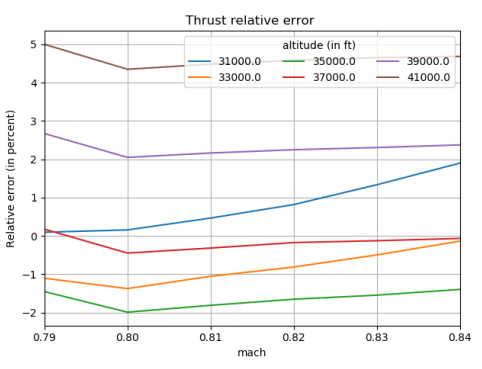

(b) Thrust relative error

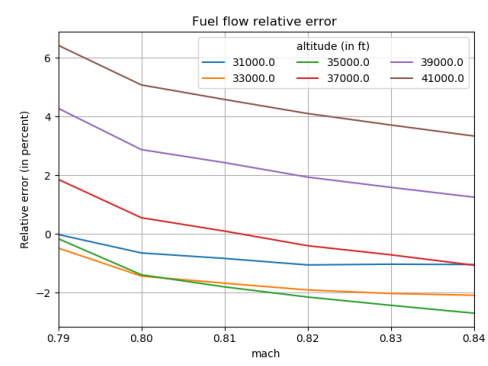

(c) Fuel flow relative error

Fig. 2 Relative errors for the specific fuel consumption $(S F C)$, the thrust and the fuel flow after propulsion calibration.

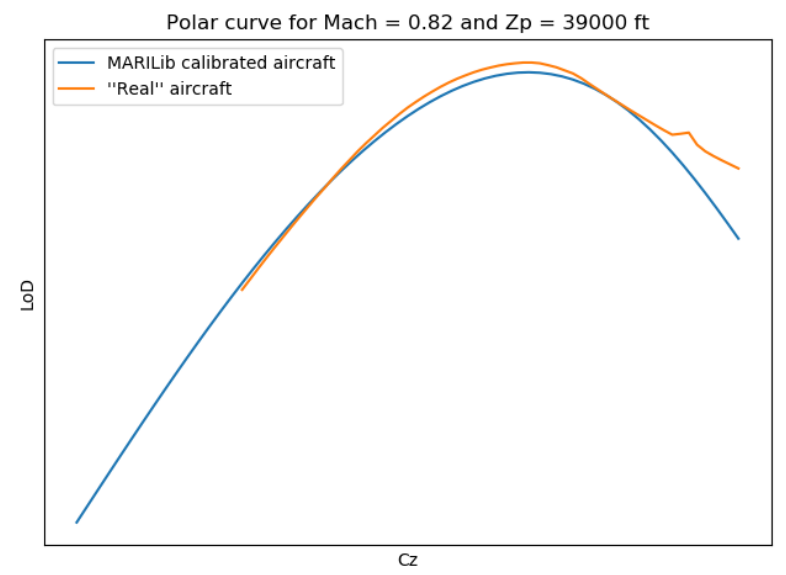

Fig. 3 Comparison of the "real" aircraft and MARILIb calibrated aircraft for Mach=0.82 and altitude $=39000 \mathrm{ft}$.

must ensure the optimum design is actually the reference aircraft. The tuning of the performance models and the adjustment of the expected performances aims at this objective. Figure 6 presents the results of this process.

As a conclusion, we have obtained an aircraft matching the reference aircraft and a calibrated MDO process that produces this aircraft and includes a set of models representing the reference aircraft. It is now possible to use it to 


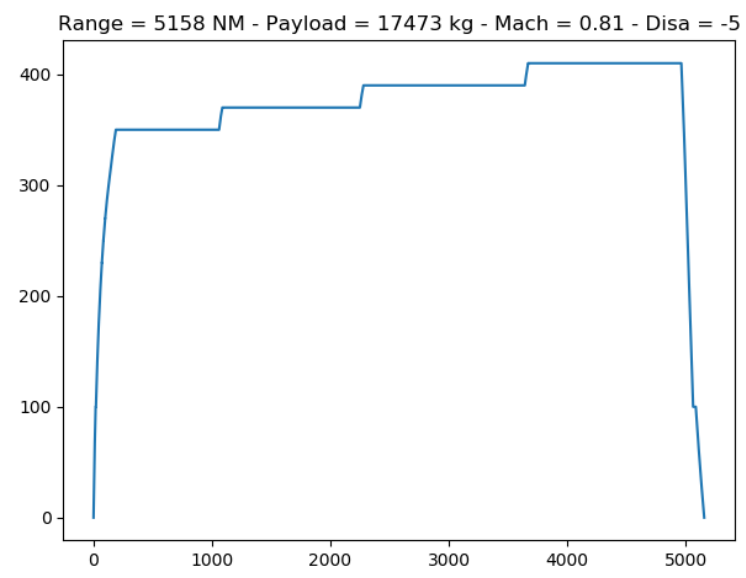

Fig. 4 Flight profile example.

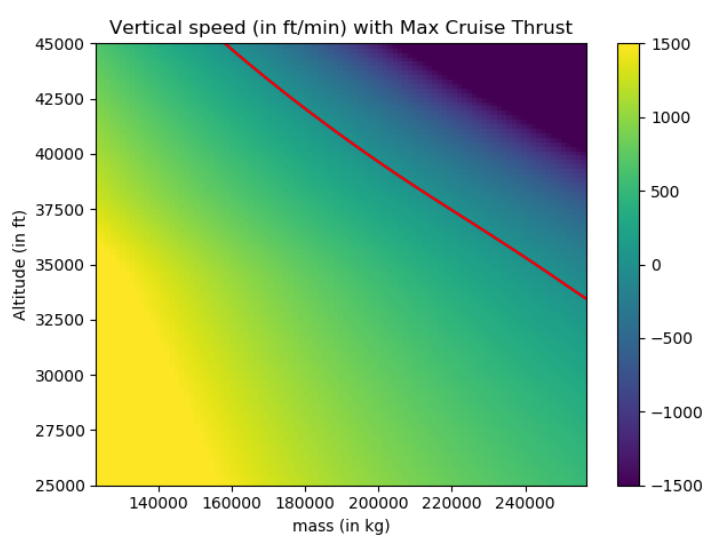

(a) Max Climb settings, vertical speed $=300 \mathrm{ft} / \mathrm{s}$

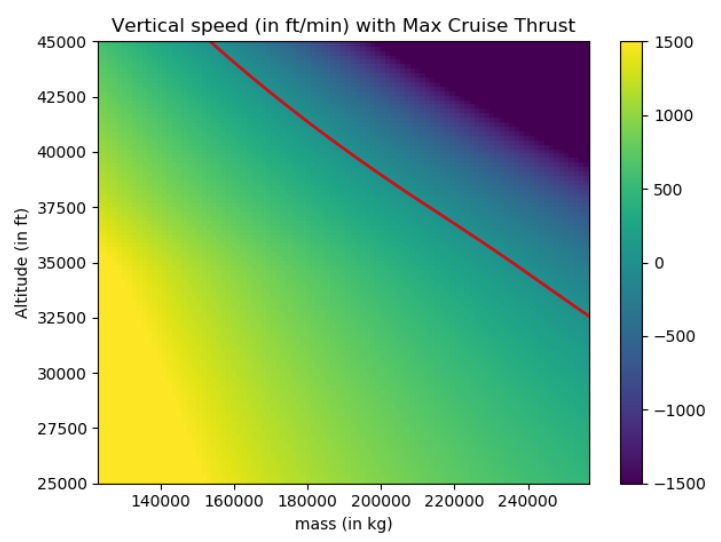

(b) Max Climb settings, vertical speed $=0 \mathrm{ft} / \mathrm{s}$

Fig. 5 Maximum vertical speed vs. mass and altitude for engine Max Climb and Max Cruise settings. Red line shows the ceiling limit corresponding to a given vertical speed.

calculate realistic flight profiles and take-off performances that we use in the rest of this paper.

\section{B. Operational data}

Observing airplane in operations is difficult as there are no database easily available that gathers all details regarding the airplane flights and the conditions in which these flights were made. The data used in this analysis are gathered in the frame of the MOZAIC project [2]. This database includes detailed flight data, from take-off to landing, for four A340-300 aircraft over a 20-year period of time. More details are available in previous work [3] from the author. However, the information of mass is not available which greatly reduces the interest of these data. To compensate this major drawback, we enrich the data with mass information for each flight. It exists different methods to estimate mass and they are adapted to the context where they are used. The one we use here relies on statistical learning approach [4].

The outcomes we want to estimate for each flight are the take-off mass (TOM) and the payload. To represent them, we note them $\mathbf{o}$. The candidate features are flight profile parameters that are available in the MOZAIC flights records. As an example, these features may include the average cruise altitude ( $\left.a l t \_c r_{a v e}\right)$, the average vertical speed during climb $\left(V z_{-} c l b_{a v e}\right)$, the highest altitude reached $\left(a l t \_c r\right.$ max $)$, but also the range (Range), the average cruise mach (Mach) or the variation of temperature compared the standard atmosphere $\left(\Delta T_{I S A}\right)$. The length of the last stage of the cruise $\left(L_{\text {last_FL }}\right)$ is added to the list of candidate features. The selection of the best set of features is described in Section III.B.3. For mathematical representation, we note the total number of features $N_{\mathbf{f}}$ and each of them is designated 


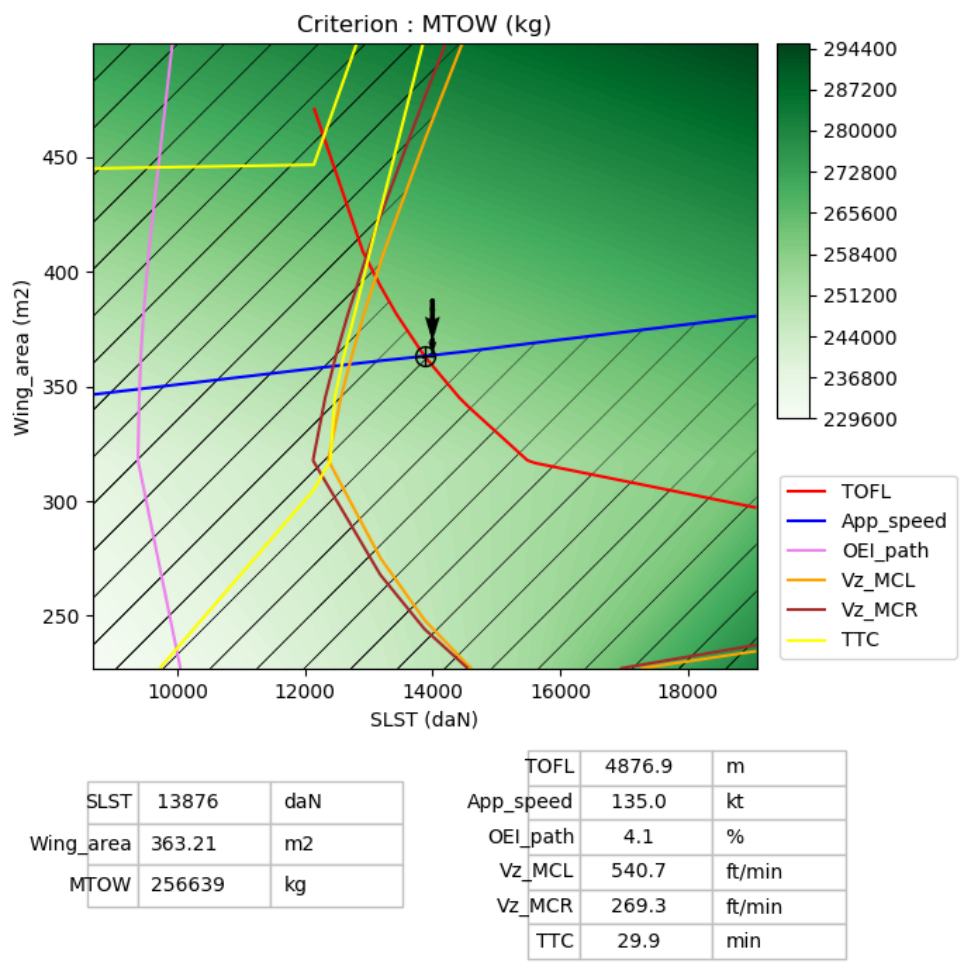

Fig. 6 Result of the optimization process after calibration.

by $\mathbf{f}_{j}, \forall j \in\left\{1 . . N_{\mathbf{f}}\right\}$. In this analysis, $N_{\mathbf{f}}=7$.

Let us call $u$ a subset of $\left\{1 . . N_{\mathbf{f}}\right\}$. The results of this process are the estimation functions presented in Equation (47).

$$
\left\{\begin{array}{l}
\text { TOW }=f\left(\mathbf{f}_{j}, j \in u_{T O W}\right) \\
\text { pld }=g\left(\mathbf{f}_{j}, j \in u_{\text {pld }}\right)
\end{array}\right.
$$

where $f$ and $g$ are two functions which will be approximated by a metamodel once the features will be defined. The method chosen belongs to the supervised learning problem category and is composed of three steps:

1) Creation of a reference database of flight records using the MARILib calibrated process presented in Section III.A. where it is possible to access to both the outcome variables and the various anticipated candidates features. It will be used to train and test the metamodels.

2) Creation of a metamodel to predict the outcome $\left(\mathbf{o} \in\{\right.$ TOW, pld $\}$ from a set of features $\left(\mathbf{f}_{j}, j \in u_{\mathbf{o}}\right)$ based on measurements made on the flight record database. Two solutions are tested: one based on linear regression and another one based on random forest [5]. The best model is then selected.

3) Application of this model on the MOZAIC data to enrich them with estimated take-off mass and payload. Note that once these two outcomes are estimated, the quantity of fuel can be calculated.

\section{Creation of a new flight profile database}

The calibration of the MARILib process enables us to calculate a large quantity of step by step missions. In this analysis, the number of flights calculated is noted $N_{M A R I L i b}$ and is equal to 4359.

In order to train the models, we want the calculated flights to cover the zone of operations of the MOZAIC flights with higher density. Five parameters characterize the mission calculation:

- the variation of temperature compared the standard atmosphere $\Delta T_{I S A}$, picked in the set $\{-5,0,5,10,15\}$,

- the cruise mach Mach, picked in the set $\{0.76,0.77,0.78,0.79,0.80,0.81,0.82,0.83,0.84\}$,

- the flight air range Range,

- the average cruise altitude $a l t \_c r_{a v e}$, and 
- the payload.

First the $\Delta T_{I S A}$ and Mach are selected together based on the 2D histogram $\mathcal{H}_{i s t_{2 D}}$ presented in Figure $7 \mathrm{a}$ and, knowing the picked values, alt_cr ave and Range are chosen based on a 2D histogram $\mathcal{H}_{i s t_{2 D \Delta T_{I S A}}, \text { Mach }}$ presented in Figure $7 \mathrm{~b}$ that depends on $\Delta T_{I S A}$ and Mach. Finally, the payload is considered as uniformly distributed between zero and the A340-300 maximum payload. Mathematically, this can be represented as indicated in Equation (5).

$$
\left\{\begin{array}{l}
\left(\Delta T_{I S A}, \text { Mach }\right) \sim \mathcal{H} \text { ist }_{2 D 1} \\
(\text { alt_cr ave }, \text { Range }) \sim \mathcal{H} \text { ist }_{2 D \Delta T_{I S A}, \text { Mach }} \\
\text { pld } \sim \mathcal{U}\left[0, \text { pld } d_{\text {max }}\right]
\end{array}\right.
$$

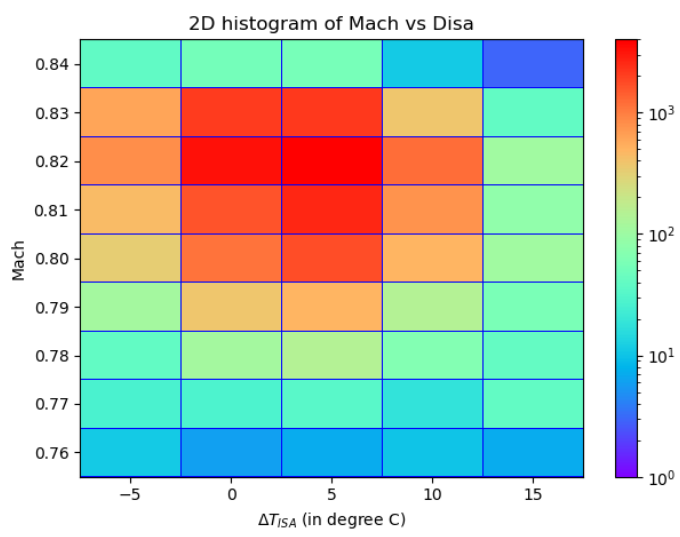

(a) $\Delta T_{I S A}$ vs. Mach

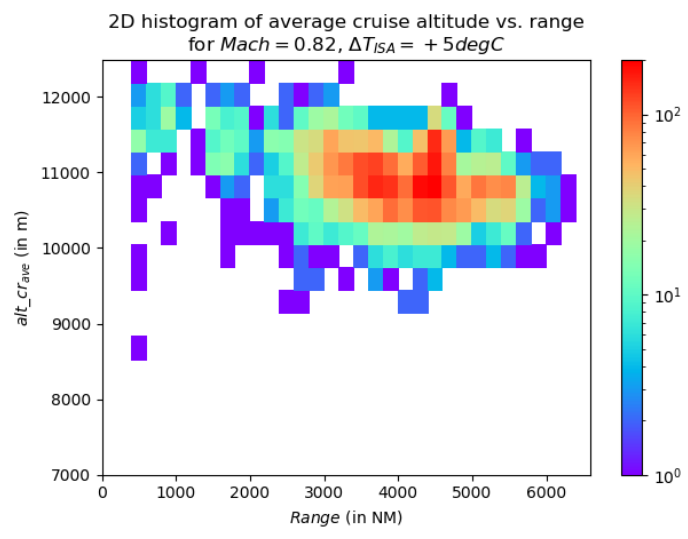

(b) Example of alt_cr ave vs. Range.

Fig. 7 2D histograms $\mathcal{H}_{i s t_{2 D 1}}$ and $\mathcal{H}{ }_{2 D} t_{2 D T_{I S A}, \text { Mach }}$ derived from the MOZAIC data.

Finally, Figure 8 illustrates the flights calculated with MARILib and compare it to the reference airplane payload-range diagram.

It is very difficult to create one single model to covert all flights and estimate the mass. The main obstacle is that the general flight profile depends on the air range. For instance very short range flights may have no cruise segment and their flight profiles differ much from the longer ones. We then decide here to split the flights in 2 groups: the first one with range flight smaller than $200 \mathrm{NM}$ and the second one with the rest of the flights.

In the rest of the analysis, we do not focus on the shortest flights. Indeed, we do not expect them to be impacted at all when modifying the take off requirements and therefore, they are not contributing much to the results of the optimization process presented in Section IV] The flight database obtained can now be used to create the metamodel to enrich the MOZAIC database with estimations of the take-off mass and the payload.

\section{Metamodeling process}

We now use the flight database obtained using MARILib to create a metamodel that enables us to obtain the functions $f$ and $g$ as described in Equation (4). These functions are intended to estimate the payload and the take-off mass based on some features of the recorded MOZAIC trajectories.

From an expert judgement, we select the seven candidate features presented in Table 1 as List 1 to create the model. The same list was used for both the payload and take-off mass metamodels. We choose the Range, the $\Delta T_{I S A}$, the alt_cr $r_{\text {ave }}$ and the Mach to represent global trajectory features and we select the $V z_{-} c l b_{a v e}$, the $a l t_{-} c r_{\text {max }}$ and the $L_{\text {last_F }} L_{L}$ to bring in the analysis information related to more specific features. The first carries information about the beginning of the flight, when the airplane mass is still very close to the take-off mass and the two others provide information about the end of the flight, when most of the fuel is burnt and the airplane mass is more closely related to the payload. The other lists presented in Table 1 are down selections of the candidates to try and test simpler metamodel. This down selection depends on the chosen outcome (either payload or take-off mass). The List 2, List 3 and List 4 presented are those identified for the take-off estimation and the random forest metamodel. The feature selection is explained in Section III.B.3. 


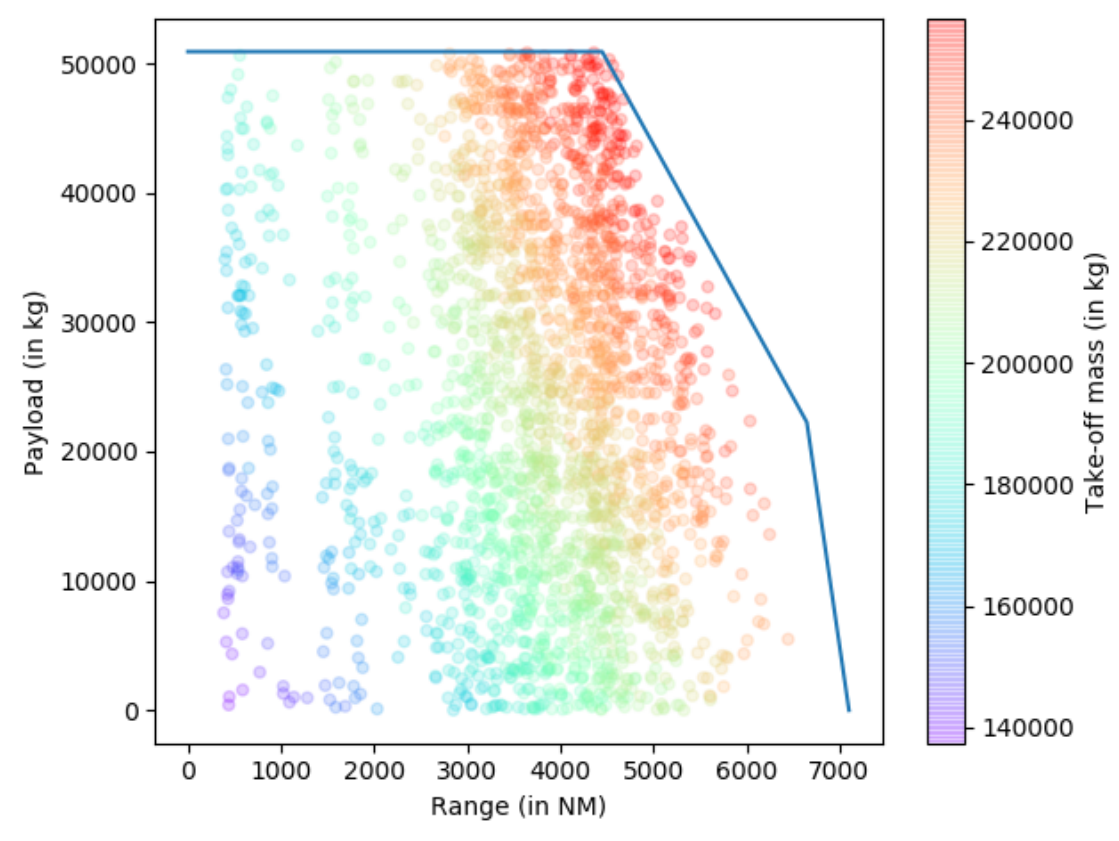

Fig. 8 Flights calculated with MARILib vs. reference aircraft payload-range diagram.

Two kind of metamodel are used in order to find the estimation functions. The first is linear regression and the second is random forest. These two metamodels have to be trained and must be tested before being used on the MOZAIC data. As there is no sample available in the MOZAIC database where the take-off mass and the payload are available, it is not possible to test the metamodel directly on MOZAIC flights. As a consequence, both the training and testing processes have to rely on the flight database created with MARILib. The MARILib flights are then split in training and testing sets. The training set contains $70 \%$ of the flights and the remaining $30 \%$ are used for testing.

\begin{tabular}{|l|l|c|c|c|c|}
\hline Features & Variable name & List 1 & List 2 & List 3 & List 4 \\
\hline Total air range & Range & $\checkmark$ & $\checkmark$ & $\checkmark$ & $\checkmark$ \\
Difference of temperature to ISA temperature & $\Delta T_{I S A}$ & $\checkmark$ & - & - & - \\
Average cruise altitude & alt_cr ave & $\checkmark$ & $\checkmark$ & $\checkmark$ & $\checkmark$ \\
Cruise mach & Mach & $\checkmark$ & $\checkmark$ & $\checkmark$ & $\checkmark$ \\
Climb vertical speed & $V z_{-}$clbave & $\checkmark$ & $\checkmark$ & $\checkmark$ & $\checkmark$ \\
Last cruise flight level & alt_cr & $\checkmark$ & \\
Length of the last cruise flight level & $L_{\text {last_FL }}$ & $\checkmark$ & $\checkmark$ & $\checkmark$ & - \\
\hline
\end{tabular}

Table 1 List of candidates features.

The analysis is made using the machine learning Python Scikit-Learn v0.21.3 library.

\section{Take-off mass estimation metamodel results}

In this section, we focus on the take-off mass estimation. Similar work is done with the payload but only the main results are presented at the end. The outcome then refers to the take-off mass.

The first step is to visualize the link between the outcome and the candidate features. Figure 9 shows the scatter plots of the take-off mass versus the candidate features. Each point represents one flight calculated with MARILib. A first interpretation shows that the take-off mass seems to be little connected to the cruise mach and the $\Delta T_{I S A}$. Stronger linear relation appears with the average cruise altitude, the average vertical climb speed and range. It also appears that some non linearity exists between the outcome and the different features. 

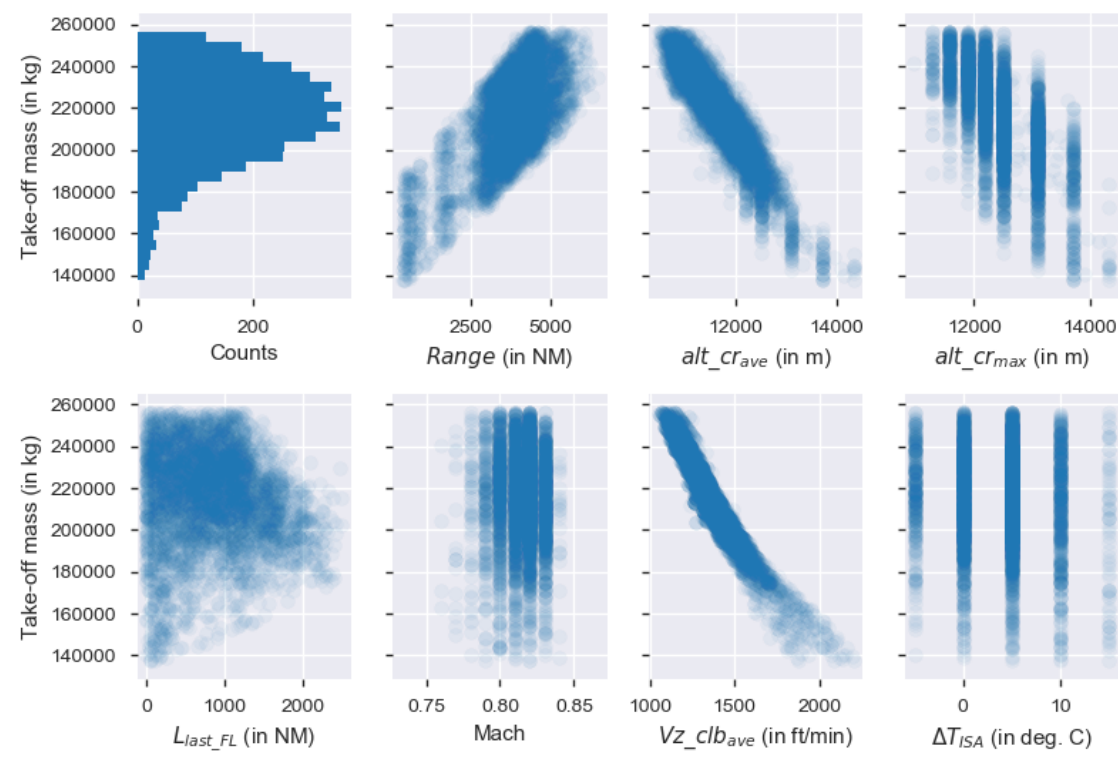

Fig. 9 Take-off mass horizontal histogram and scatter plots of the Take-off mass vs. the candidate features.

$$
\mathbf{o}=c_{0}+\sum_{j \in u_{\mathbf{o}}} c_{j} \mathbf{f}_{j}
$$

A first metamodel is created based on linear regression model described in Equation [6] and the training set presented in Section III.B.2 The results are presented in Table2 2 For each candidate feature, it gives the corresponding regression coefficient, noted $c_{j}, \forall j \in u_{T O W}$. It also provides an evaluation of the performance of the linear regression through the mean absolute error $(M A E)$, the mean squared error $(M S E)$ and $(R M S E)$, the accuracy and the $R^{2}$ score. These performance indicators are calculated based on the test set presented in Section III.B.2. The smaller the errors, the better the model. Concerning the accuracy and the $R^{2}$ score, the closer to one, the better the model.

\begin{tabular}{|c|c|c|}
\hline Features & Regression coefficients & Performance metrics \\
\hline Intercept $\left(c_{0}\right)$ & $8.2910^{5}$ & \\
\hline Range & $3.9910^{-3}$ & $M A E=1566.72 \mathrm{~kg}$ \\
\hline alt_cr ave & $-1.8810^{1}$ & $M S E=5.74010^{6} \mathrm{~kg}^{2}$ \\
\hline alt_cr $r_{\max }$ & -6.65 & $R M S E=2395.88 \mathrm{~kg}$ \\
\hline$L_{\text {last_FL }}$ & $-1.9910^{-3}$ & Accuracy $=99.222 \%$ \\
\hline Mach & $-3.7710^{5}$ & $R^{2}=0.9895$ \\
\hline$\Delta T_{I S A}$ & $-6.4110^{1}$ & \\
\hline$V z_{-} c l b_{\text {ave }}$ & $-4.0210^{3}$ & \\
\hline
\end{tabular}

Table 2 Take-off mass linear regression model and errors.

The sign of the regression coefficients appears realistic. On one hand, if the range increases, we actually expect the take-off mass to increase as well. On the other end, it appears normal that the take-off mass reduces if the average cruise altitude, the highest cruise altitude and the last flight level length increase. However, the results for cruise mach and the ambient temperature are more surprising. Indeed, if the mach increases higher fuel consumption is expected and if the temperature increases, lower propulsion performances are expected which both should result for a given mission in higher take-off mass.

Due to the non-linear relations observed between the take-off mass and the different features in Figure 9, we test another regression method by using a random forest. This technique is a good candidate for non-linear problem as 
it is easy to calibrate and its outputs are easy to interpret [4]. The parameters we use to tune the random forest are the number of trees, the maximum depth, the maximum number of features selected for creating each tree and the minimum number of samples in each leaf. In order to simplify the model, we also evaluate the importance of each candidate feature and keep those having an impact on the results. The performance of the random forest is evaluated using the same indicators as for the linear regression. However, in order to evaluate the quality of the random forest, the out-of-bag score (OOB score) is also calculated. A total of ten analysis are made to choose the best parameters of the random forest metamodel. Furthermore, the random forest regression also provides importance score for each feature and therefore enables to classify them. Three additional analysis are then made to try and simplify the model by removing the features having the smallest importance. The performances are presented in Table 3 The importance of each candidate feature is presented in Table 4

\begin{tabular}{|c|c|c|c|c|c|c|c|c|c|c|c|}
\hline Analysis & $\begin{array}{c}\text { Tree } \\
\text { number }\end{array}$ & $\begin{array}{c}\text { Max } \\
\text { depth }\end{array}$ & $\begin{array}{c}\text { Mini- } \\
\text { mum leaf } \\
\text { samples }\end{array}$ & $\begin{array}{c}\text { Max } \\
\text { fea- } \\
\text { tures }\end{array}$ & $\begin{array}{c}\text { Feature } \\
\text { List }\end{array}$ & $\begin{array}{c}M A E \\
\text { in kg) }\end{array}$ & $M S E$ & $\begin{array}{c}R M S E \\
\text { (in kg) }\end{array}$ & Accuracy & $R^{2}$ & $\begin{array}{c}\text { OOB } \\
\text { score }\end{array}$ \\
\hline 1 & 100 & 3 & 10 & 3 & List 1 & 4417 & $31.1010^{6}$ & 5576 & 97.92 & $94.30 \%$ & $93.88 \%$ \\
2 & 100 & 10 & 10 & 3 & List 1 & 2249 & $8.3710^{6}$ & 2894 & 98.93 & $98.47 \%$ & $98.30 \%$ \\
3 & 100 & 15 & 10 & 3 & List 1 & 2228 & $8.2310^{6}$ & 2868 & 98.94 & $98.49 \%$ & $98.33 \%$ \\
4 & 200 & 15 & 10 & 3 & List 1 & 2221 & $8.2010^{6}$ & 2863 & 98.94 & $98.50 \%$ & $98.37 \%$ \\
5 & 500 & 15 & 10 & 3 & List 1 & 2243 & $8.3110^{6}$ & 2884 & 98.93 & $98.48 \%$ & $98.36 \%$ \\
6 & 200 & 15 & 5 & 3 & List 1 & 1908 & $6.2910^{6}$ & 2508 & 99.09 & $98.85 \%$ & $98.75 \%$ \\
7 & 200 & 15 & 15 & 3 & List 1 & 2423 & $9.5710^{6}$ & 3094 & 98.85 & $98.25 \%$ & $98.06 \%$ \\
8 & 200 & 15 & 5 & 4 & List 1 & 1888 & $6.1710^{6}$ & 2484 & 99.10 & $98.87 \%$ & $98.77 \%$ \\
9 & 200 & 15 & 5 & 5 & List 1 & 1897 & $6.2510^{6}$ & 2500 & 99.09 & $98.85 \%$ & $98.74 \%$ \\
10 & 200 & 15 & 5 & 6 & List 1 & 1918 & $6.3510^{6}$ & 2520 & 99.08 & $98.84 \%$ & $98.72 \%$ \\
11 & 200 & 15 & 5 & 4 & List 2 & 1911 & $6.2910^{6}$ & 2508 & 99.09 & $98.85 \%$ & $98.74 \%$ \\
12 & 200 & 15 & 5 & 4 & List 3 & 1897 & $6.1710^{6}$ & 2483 & 99.09 & $98.87 \%$ & $98.76 \%$ \\
13 & 200 & 15 & 5 & 4 & List 4 & 1937 & $6.2610^{6}$ & 2503 & 99.07 & $98.85 \%$ & $98.72 \%$ \\
\hline
\end{tabular}

Table 3 Performances of the take-off mass random forest regression model for different combination of settings.

The first result of the random forest process is that the parameters that minimize the error and maximize the accuracy, the $R^{2}$ and the OOB score is the one related to analysis 8 as presented in Table 3 . We observe that the total number of candidate features is $N_{\mathrm{f}}=7$ and that the recommended maximum number of features to be taken for growing the trees is around one third of $N_{\mathbf{f}}$ [4]. This is why we started the analysis with a maximum number of features for creating the forest regression trees equal to 3 . This parameter can nevertheless be tuned depending of the case considered [4]. Increasing this number up to 4 improved the performance of the metamodel and reduced both the MAE and MSE while increasing the accuracy, $R^{2}$ score and the OOB score. Increasing it more $(5$ or 6$)$ reduced the performances.

The random forest metamodel also provides importance scores that enable to classify the features. Table 4 shows these scores for the thirteen analysis. The random forest classification confirms the judgement of expert on the first selection of the candidate features. Information related to the climb vertical speed, the average cruise altitude and the range appears the most important, the climb conditions exhibiting at least more than half of the total importance. The features of the end of the cruise bring little information. Surprisingly, the cruise mach and the temperature have little importance scores on this estimation. In list 2, the temperature information is removed of the features used to predict the outcome and we can see that this does not improve the performance of the regression metamodel. In list 3, when both the temperature information and the length of the last cruise flight level are removed, the performance of the model increases. Removing other features leads to lower performances.

As a result, we retain analysis 8 settings as the ones providing the best results:

- Number of features: 7 (presented in the Table 1 )

- Maximum features: 4

- Tree number: 200 (increasing to 500 does not improve much the results)

- Max depth: 15 


\begin{tabular}{|c|c|c|c|c|c|c|c|}
\hline Analysis & 1 & 2 & 3 & 4 & 5 & 6 & 7 \\
\hline$V z_{\text {_clb }}$ ave & $54.08 \%$ & $53.21 \%$ & $53.15 \%$ & $52.05 \%$ & $53.85 \%$ & $52.69 \%$ & $51.70 \%$ \\
alt_crave & $27.08 \%$ & $26.94 \%$ & $27.27 \%$ & $27.81 \%$ & $26.93 \%$ & $27.10 \%$ & $27.77 \%$ \\
Range & $11.37 \%$ & $12.67 \%$ & $12.22 \%$ & $13.02 \%$ & $12.47 \%$ & $13.05 \%$ & $13.50 \%$ \\
alt_cr max & $6.96 \%$ & $5.50 \%$ & $5.56 \%$ & $5.38 \%$ & $5.03 \%$ & $5.17 \%$ & $5.45 \%$ \\
$L_{\text {last_FL }}$ & $0.36 \%$ & $0.54 \%$ & $0.60 \%$ & $0.59 \%$ & $0.57 \%$ & $0.67 \%$ & $0.59 \%$ \\
Mach & $0.15 \%$ & $1.11 \%$ & $1.17 \%$ & $1.13 \%$ & $1.12 \%$ & $1.26 \%$ & $0.98 \%$ \\
$\Delta T_{I S A}$ & $0.01 \%$ & $0.02 \%$ & $0.03 \%$ & $0.03 \%$ & $0.03 \%$ & $0.06 \%$ & $0.02 \%$ \\
\hline Analysis & 8 & 9 & 10 & 11 & 12 & 13 & \\
\hline$V z_{\text {_clb ave }}$ & $60.69 \%$ & $72.61 \%$ & $83.08 \%$ & $70.62 \%$ & $77.99 \%$ & $93.59 \%$ & \\
alt_cr ave & $27.55 \%$ & $21.45 \%$ & $14.09 \%$ & $23.86 \%$ & $18.69 \%$ & $4.15 \%$ & \\
Range & $8.57 \%$ & $4.28 \%$ & $1.61 \%$ & $3.82 \%$ & $2.20 \%$ & $1.31 \%$ & \\
alt_cr max & $1.79 \%$ & $0.45 \%$ & $0.10 \%$ & $0.48 \%$ & $0.13 \%$ & - & \\
$L_{\text {last_F }}$ & $0.25 \%$ & $0.18 \%$ & $0.16 \%$ & $0.19 \%$ & - & - & \\
Mach & $1.12 \%$ & $1.01 \%$ & $0.94 \%$ & $1.02 \%$ & $0.98 \%$ & $0.96 \%$ & \\
$\Delta T_{I S A}$ & $0.03 \%$ & $0.02 \%$ & $0.02 \%$ & - & - & - & \\
\hline
\end{tabular}

Table 4 Relative importance of each candidate features in the random forest analysis presented in Table 3

- Minimum leaf samples: 5

The mean absolute error $(M A E) 1888 \mathrm{~kg}$ which corresponds to 19 passengers, which is reasonable. However, the mean squared error $(M S E)$, which is more sensitive to large errors than the $M A E$, shows some variance in the errors as the square root of the $M S E$ is $2484 \mathrm{~kg}$.

Similar work was done on the payload estimation. The performance of the metamodel was not as good and the $M A E$ was found equal to $2172 \mathrm{~kg}$. It is important to note that in this case, the features with the highest importance were those related to the highest cruise altitude, the average cruise altitude and the length of the last cruise flight level. The information related to the last part of the flight carried more information as the judgement of expert anticipated. The best settings were found to be the following:

- Maximum features: 5

- Tree number: 200 (increasing to 500 does not improve much the results)

- Max depth: 15

- Minimum leaf samples: 5

When comparing the linear regression and the random forest, we observe that the linear regression performs better than the random forest although we expected the opposite as some non-linearity exists between the outcome and the features. An attempt to use the linear regression metamodel on the MOZAIC flights revealed a strong tendency to over estimate the take-off mass maybe due to extrapolation. No further investigation are made on this topic in the frame of this paper.

An interesting characteristic of the random forest is that there is no risk of extrapolation which ensures the predicted outcome to fall in the anticipated range of values. As a consequence, we choose the random forest metamodel described above to predict the take-off mass for each flight or the MOZAIC database.

\section{Application of the model to the MOZAIC database enrichment}

The mass is the key information that is missing in the MOZAIC database. This database contains more than 30000 flights records over a period of 20 years for the A340-311 which is the reference airplane of this paper. After having pre-processed this database, we select 27520 flights. In Section III.B.3, we create a metamodel based on random forest method. It enables to estimate the take-off mass based on flight profile features, among which the three most important are the $V z_{-} c l b_{a v e}$, the $a l t \_c r_{a v e}$ and the Range. We apply now this model to the MOZAIC database flights. We 
focus our analysis on flights longer than $200 \mathrm{NM}$. The histogram of the estimated take-off mass is shown in Figure 10a Estimating both the take-off mass and the payload from the metamodels created in Section III.B.3 may not result in consistent results as these two parameters are linked together. The process we use here is divided in three steps:

1) For each flight of the MOZAIC database, we also enrich the database with the departure airport, its altitude and the longest runway available for take-off. Knowing the temperature from MOZAIC record and using MARILib take-off model, we calculate for each flight the maximum take-off mass for the given take-off field length, altitude and temperature. This maximum take-off mass is smaller or equal to the reference aircraft MTOW. The final take-off mass retained is the smallest of the one estimated with the random forest model and the maximum take-off mass calculate.

2) Knowing the take-off mass and the range for each flight, we calculate the on-board mass (mass on board) using MARILib mission module. This module calculates the block fuel and the total fuel ( fuel $\left._{\text {total }}\right)$ for given take-off mass, range, and flying conditions. The total fuel then includes both the block fuel and some regulatory reserves in case of diversion from the destination airport. The on-board mass corresponds to the sum of the payload and some extra fuel fuel extra that is not burnt during flight nor part of the reserves. This extra fuel may also account for additional reserves for en-route diversion or additional fuel the airline chooses to carry during flight. Using

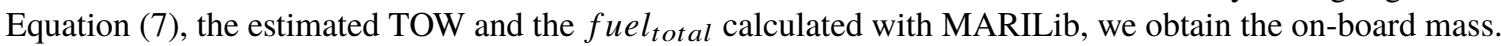

$$
\left\{\begin{array}{l}
\text { TOW }=O W E+\text { pld }+ \text { fuel }_{\text {on board }} \\
\text { TOW }=O W E+\text { mass }_{\text {on board }}+\text { fuel }_{\text {total }} \\
\text { TOW }=O W E+\text { pld } \text { fuel }_{\text {extra }}+\text { fuel }_{\text {total }}
\end{array}\right.
$$

3) The on-board mass is compared to the reference airplane maximum payload.

- If the on-board mass is bigger, we consider the airplane carries extra mass equal to the difference between the on-board mass and the maximum payload. This is illustrated in Equation (8). We then consider the payload to be equal to the maximum payload.

$$
\text { fuel }_{\text {extra }}=\text { mass }_{\text {on board }}-\text { pld }_{\text {max }}
$$

- If the on-board mass is smaller than the maximum payload, we consider no extra fuel is carried.

The histogram of the estimated payload is presented in Figure $10 \mathrm{~b}$ It shows a peak at the maximum payload which can either show that the airline has flown as much as possible with the maximum payload or be a limit of the current process to estimate the mass. Figure 11 shows a comparison of the payload-range diagram of the reference airplane (calculated for and altitude of $35000 \mathrm{ft}$ ) and the estimated payload and take-off mass. It shows a good consistency of the estimated payload and take-off mass.

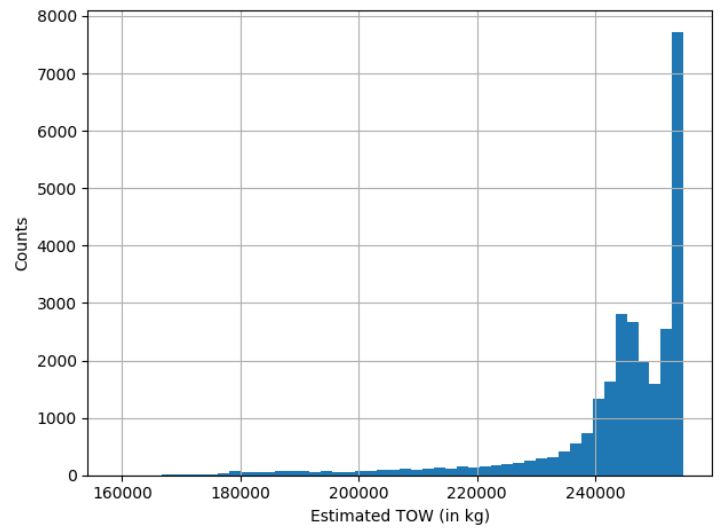

(a) MOZAIC estimated take-off mass.

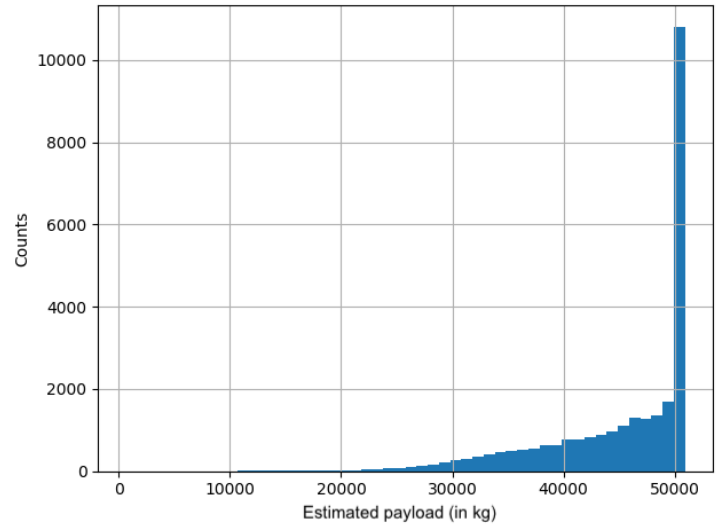

(b) MOZAIC estimated payload

Fig. 10 Histogram of the MOZAIC estimated take-off mass (left) and estimated payload (right). 


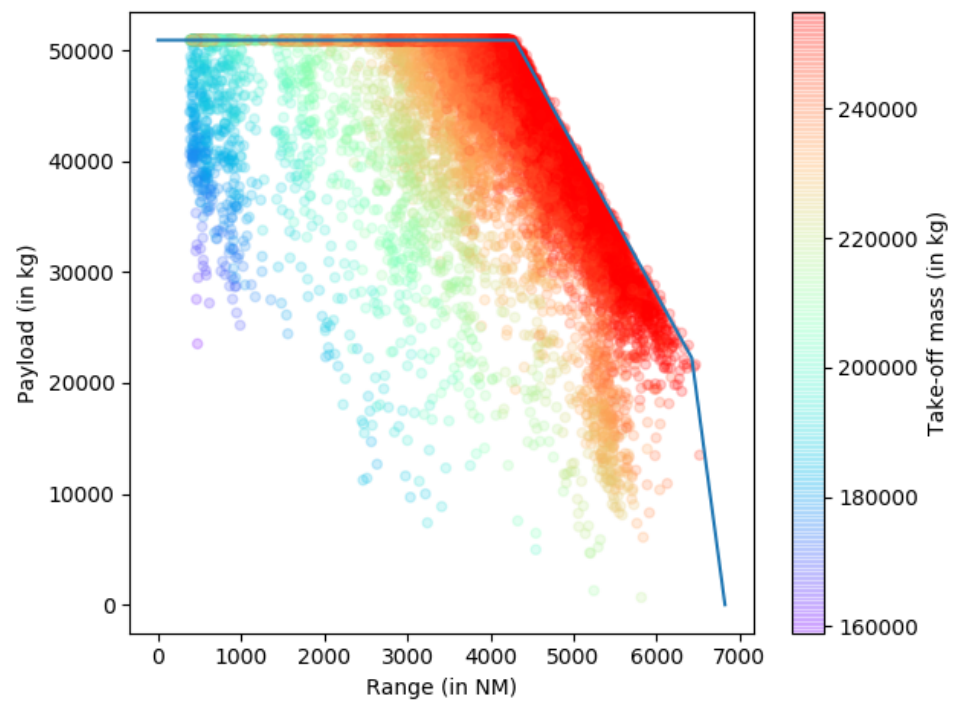

Fig. 11 Payload-range diagram of the reference airplane (altitude $=35000 \mathrm{ft}$ ) with the MOZAIC estimated payload and take-off mass.

\section{Conclusion}

The Section [II] tackles the question of the airplane design tools and operational data. The operational MOZAIC data brings us to choose a specific reference aircraft (the A340-311) and to tune the generic MARILib models in order to make it specific to this reference airplane. Then the missing information of mass in the MOZAIC flight records required us to estimate both the take-off mass and the payload using machine learning methods. We finally obtain an enriched database with estimated mass information. It is now possible to challenge the take-off requirements by confronting them to more realistic conditions.

\section{Take-off requirements challenging method}

The take-off requirements are usually taken into account in the optimization process as constraints. Many parameters characterise these requirements. Among them, we find the take-off mass, the take-off field length, the pressure altitude of the airport, the local temperature, the wind direction and intensity, and the runway condition, either wet or dry. MARILIb take-off performance models do not take into account wind or runway conditions. Therefore these parameters are not considered in this work. Noting that the requirements are stated for maximum take-off mass (MTOM), then the list of the parameters considered reduces to:

- the take-off field length (TOFL),

- the pressure altitude of the airport (altp),

- the local temperature or more precisely its difference with the standard temperature $\left(\Delta T_{I S A}\right)$.

In this part, we first define a process to calculate the impact of take-off requirements on operational costs. In a second time, we classify the impact of each take-off parameter on the operational costs. Third, we create a model to estimate the consequences of alleviating the take-off requirements on the operational costs. Finally, we propose a new MDAO process to consider take-off parameters as design variables rather than constraints. The results are presented in the next part.

\section{A. Process to calculate the impact of take-off requirements on operational costs}

The process used for the calculation is presented in Figure 12

The MDAO process is the core of the general process and is based on MARILib. It consists in a constrained optimization process following a multidisciplinary feasible (MDF) formulation. This minimization problem is 


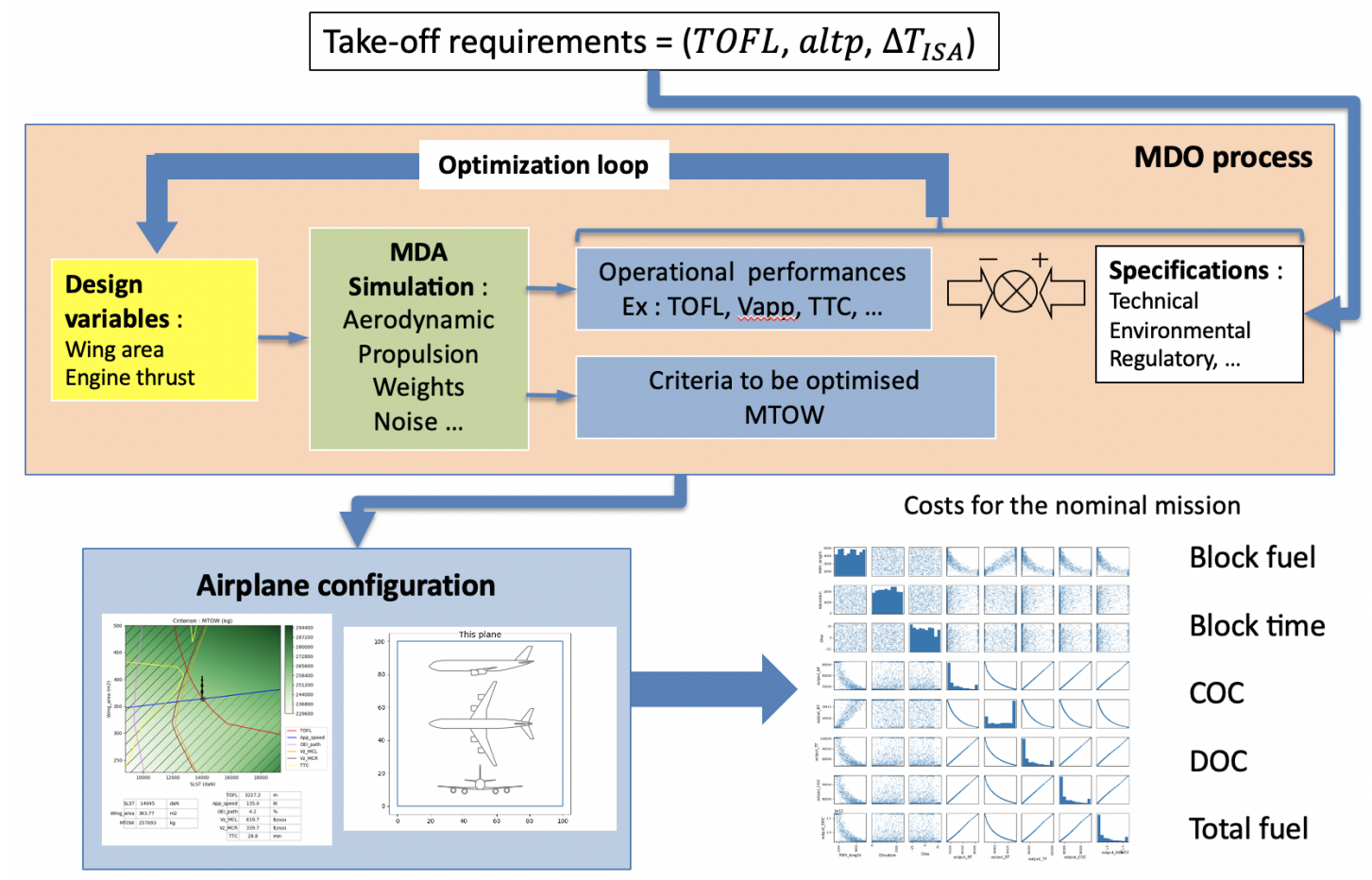

Fig. 12 General process to calculate take-off requirements impact on operational costs.

summarized in Equation 9

$$
\begin{array}{ll}
\min _{x} & f(x) \\
\text { s.t. } & g(x) \geq 0
\end{array}
$$

where:

- $x$, the design variable vector, includes the wing reference area wing_area and the engine sea level static thrust $(S L S T)$

- $f(x)$, the objective function can be the $M T O W$, the fuel burn, the cash operating cost $C O C$, the direct operating cost $D O C$ or another value of interest.

- $g(x)$, the inequality constraint vector, includes the take-off field length (TOFL), the approach speed (App_speed), the one-engine-inoperative path after take-off $\left(O E I_{-}\right.$path $)$, the climb and cruise ceiling vertical speeds $\left(V z_{-} M C L\right.$ and $\left.V z_{-} M C R\right)$ and the time-to-climb to cruise altitude (TTC).

The take-off requirements parameters (TOFL, altp, $\left.\triangle T_{I S A}\right)$ are set at the beginning of the design process and the MDAO process is run. The objective function and the constraints are both non-linear. The optimization algorithms used are the sequential least squares programming algorithm (SLSQP) or the trust region algorithm (trust-const) of Python Scipy library. Using the MARILib tool calibrated in Section III.A. Figure 13 shows the result of an optimization run where the objective function is the $C O C$. The constraints have the following values:

- Take-off constraint: $T O F L \leq 4877 \mathrm{~m}$, considering altp $=5431 \mathrm{ft}, \Delta T_{I S A}=+25 \mathrm{deg}$. They correspond to the Denver airport conditions, which have the reputation to be severe.

- Approach speed : App_speed $\geq 135 \mathrm{kts}$

- One-engine-inoperative path after take-off: $O E I_{-}$path $\geq 1.6 \%$

- Climb ceiling vertical speed: $V z_{-} M C L \geq 300 \mathrm{ft} / \mathrm{min}$

- Cruise ceiling vertical speed: $V z_{-} M C R \geq 0 \mathrm{ft} / \mathrm{min}$

- Time-to-climb to cruise altitude: $T T C \leq 60 \mathrm{~min}$

The output of this process is an airplane configuration. The process also provides the costs for the design cost mission. The mission module is available in MARILib and relies on Breguet equation. 


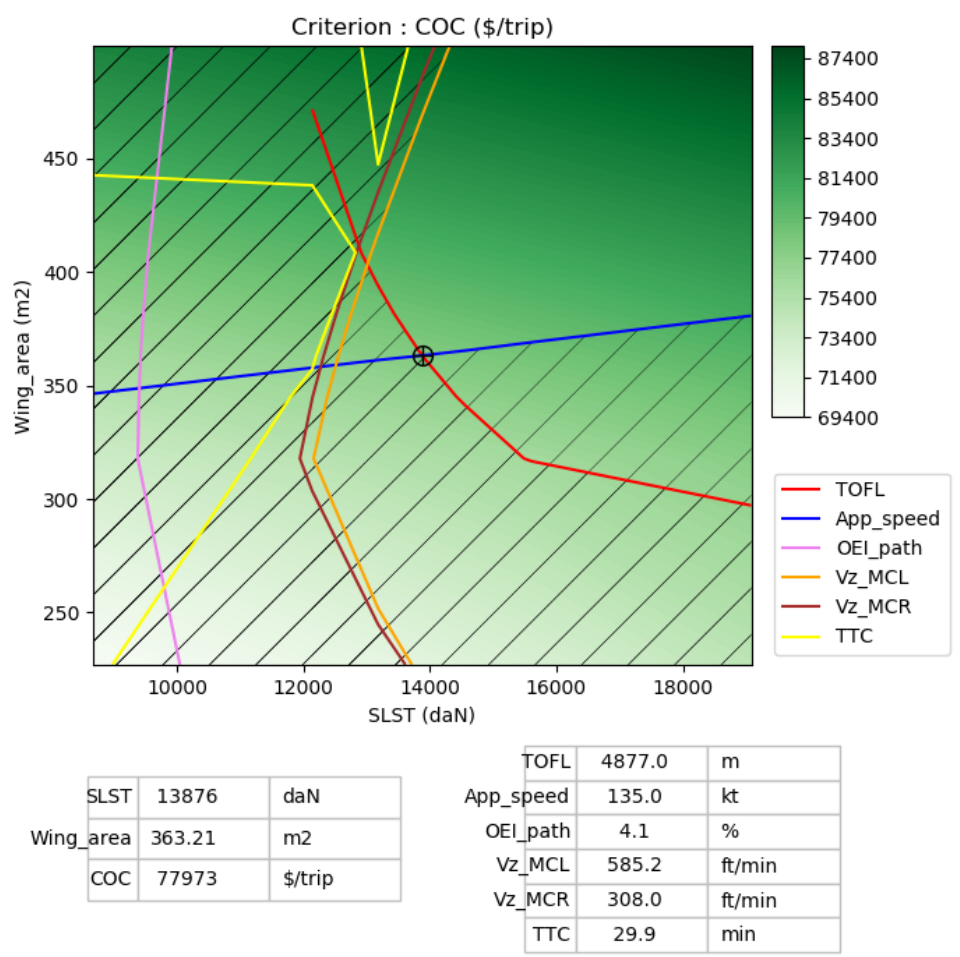

Fig. 13 Example of the result of an optimization run using SLSQP algorithm for the reference set of take-off parameters.

\section{B. Sensitivity analysis}

The aim of this section is to study the sensitivity of the quantity of interest with respect to the take-off requirements parameters using a global sensitivity analysis. Two types of sensitivity indices will be considered here: first, the classical Sobol indices that are variance based and then a generalized index taking into account the whole distribution of the quantity of interest. On this purpose, we calculate Sobol indices [6] and classify the impact of the take-off requirements parameters (TOFL, altp, $\triangle T_{I S A}$ ) on the operational costs. The quantities of interest considered here are the following costs: the block fuel, the block time, the cash operating cost (COC), the direct operating cost (DOC), and the total fuel. As we can obtain these costs at different moment in the process, we compare the results obtained in each case. Table 5 presents the set of scalar costs considered in this analysis. Sobol indices are variance based indices. This

\begin{tabular}{|l|c|}
\hline Operation considered & Design cost mission \\
\hline Cost processing & One-flight cost \\
\hline & block fuel \\
block time \\
Cost considered & COC \\
& DOC \\
& total fuel \\
\hline Sensitivity indices & Sobol and CVM \\
\hline Calculation method & PCE and P\&F \\
\hline
\end{tabular}

Table 5 The set of costs considered and the associated sensitivity indices.

means they focus only on the variance and decompose it in order to measure how much each input variable impacts the outcome variance. Even if Sobol indices can be extended to multivariate output [7], they are commonly used on scalar 
outputs. Other sensitivity indices, different from the Sobol indices, also exist. They generalize Sobol indices not only for multivariate outputs [8] but also for the whole output distribution. The index proposed relies on the Cramér von Mises (CVM) distances.

Sensitivity indices can be estimated using direct or quasi-Monte Carlo simulations but this usually requires a large number of evaluations which can make it impractical. For scalar output, other ways exists and the one proposed by Blatman and Sudret [9] uses Polynomial Chaos Expansion (PCE). Knowing the distribution of the input parameters, adequate families of polynomials can be chosen to decompose the output distribution. The coefficients of the PCE enable to obtain the value of the corresponding Sobol indices. However, for more general sensitivity analysis no such tool exists as the PCE approach. As indicated in [10], other estimators exist based on the Pick and Freeze (P\&F) approach. It relies on the fact that it is less computationally challenging to estimate the co-variance between a random variable and its rather than the variance of a conditional expectation.

Table 6 presents the results obtained for the two index (Sobol and $C V M$ ). They are calculated using PCE and $P \& F$ methods. It is important to underline that these results show only the direct effect of the inputs on the outputs. The combined influence of the inputs on the outputs are not shown and are not covered in this analysis. The results presented are calculated on a design of experiments (DOE) containing 1000 points uniformly distributed in the following ranges:

- for the TOFL, between 1500 and 5000 meters,

- for the altp, between 0 and 2500 meters,

- for the $\Delta T_{I S A}$, between -30 and +30 Celsius degrees.

\begin{tabular}{|c|c|c|c|c|c|}
\hline \multirow{2}{*}{\multicolumn{2}{|c|}{$\begin{array}{c}\text { Operation considered } \\
\text { Calculation method }\end{array}$}} & \multicolumn{4}{|c|}{ Design cost mission } \\
\hline & & PCE & & & $P \& F$ \\
\hline \multicolumn{2}{|c|}{ Sensitivity indices } & \multicolumn{2}{|c|}{ Sobol } & CVM & CVM Multivariate \\
\hline Output names & Input names & \multicolumn{4}{|c|}{ Index values } \\
\hline \multirow{3}{*}{ Block fuel } & TOFL & $81.36 \%$ & $70.70 \%$ & $55.90 \%$ & \multirow{15}{*}{$\begin{array}{l}41.34 \% \\
23.82 \% \\
29.98 \%\end{array}$} \\
\hline & altp & $6.25 \%$ & $13.48 \%$ & $9.95 \%$ & \\
\hline & $\Delta T_{I S A}$ & $6.10 \%$ & $14.75 \%$ & $18.75 \%$ & \\
\hline \multirow{3}{*}{ Block time } & TOFL & $79.09 \%$ & $67.54 \%$ & $40.01 \%$ & \\
\hline & altp & $7.41 \%$ & $12.44 \%$ & $6.48 \%$ & \\
\hline & $\Delta T_{I S A}$ & $8.71 \%$ & $18.30 \%$ & $9.47 \%$ & \\
\hline \multirow{3}{*}{$\mathrm{COC}$} & TOFL & $81.36 \%$ & $70.70 \%$ & $55.90 \%$ & \\
\hline & altp & $6.25 \%$ & $13.48 \%$ & $9.95 \%$ & \\
\hline & $\Delta T_{I S A}$ & $6.10 \%$ & $14.75 \%$ & $18.75 \%$ & \\
\hline \multirow{3}{*}{ DOC } & TOFL & $81.38 \%$ & $70.73 \%$ & $55.90 \%$ & \\
\hline & altp & $6.25 \%$ & $13.49 \%$ & $9.95 \%$ & \\
\hline & $\Delta T_{I S A}$ & $6.10 \%$ & $14.79 \%$ & $18.75 \%$ & \\
\hline \multirow{3}{*}{ Total fuel } & TOFL & $81.63 \%$ & $70.76 \%$ & $55.90 \%$ & \\
\hline & altp & $5.94 \%$ & $13.41 \%$ & $9.95 \%$ & \\
\hline & $\Delta T_{I S A}$ & $5.81 \%$ & $14.55 \%$ & $18.75 \%$ & \\
\hline
\end{tabular}

Table 6 Sensitivity indices for the different costs related to the design cost mission.

The first conclusion that we can draw is that all the indices present consistent classification of the three inputs, for all the outputs consid. The take-off field length has by far the greatest influence on the outputs variability. The altitude and the temperature have smaller impact. The Sobol index shows that the outputs are equally sensitive to these two parameters. The Cramér von Mises indices indicate that the temperature variability influences more the outputs. The multivariate $C V M$ index shows similar results with respect to the temperature although it shows that the altp and $\Delta T_{I S A}$ have a significantly increased influence on the output vector. This difference may come from the fact that $C V M$ index takes into account the full distribution of the output and does not focus on the variance as the Sobol index. Concerning the $C V M$ index on the scalar outputs the sum of the three index goes down to $85 \%$ (and even 55\%) for 
the the block time), which may be related shifting a part of the individual influence of each input to higher combined influence. The two methods used to estimate the Sobol index show similar results even if the one using $P \& F$ shows greater importance for both altp and $\Delta T_{I S A}$. Apart from the block time, the indices calculated are very close from one output to another. The block time shows different results. This may be explained by the fact that the duration of the flight does not depend on take-off requirement as much as the other four outputs. To conclude, we note that the take-off field length has the greatest impact on the output. For the rest of the analysis, we therefore keep only the $T O F L$ to specify the take-off requirements and will set the design departure airport altitude and temperature to the following reference values: altp $=0 f t$ and $\Delta T_{I S A}=0 \operatorname{deg} C$.

\section{Model for estimating the impact on costs of reduced take-off performances}

When reducing the take-off requirements stringency, the design process generates an airplane with reduced take-off performances. This means that for a given take-off mass and given conditions (airport altitude or temperature), the required take-off field length for the designed airplane will be increased. However, the existing runways will not be extended to accommodate the new airplane. This implies that, in some situations, the maximum take-off mass from a departure airport will be reduced. As we consider that the flight program is unchanged, the ranges the airplane will have to fly will remain the same and the reduction of the take-off mass will imply a reduction in the payload. This will induce some income loss as the airline will not be able to meet the demand and may trigger some additional costs as the airline may have to give compensation to passengers not taken on board. We can even imagine that severe reduction of take-off performances even lead to the cancellation of the flight.

The model we use in this analysis addresses these considerations in order to estimate a cost of reduced take-off performances for the airlines. We consider a very simple model where we make the assumption that the cost for the airlines is proportional the reduction in the payload mass. The Equation (10] represents this model.

$$
C_{f l t}=c_{p l d} \Delta p l d_{f l t}
$$

where:

- $\Delta$ pld $_{f l t}$ the reduction in payload for one flight, expressed in $\mathrm{kg}$,

- $c_{\text {pld }}$ the cost related to the disembarking of one kilogram of payload (either freight or passenger),expressed in $\$ / k g$ and

- $C_{f l t}$ the additional cost for one flight due the reduction of the take-off performance, expressed in $\$$.

Considering the full flight program $\mathcal{P}$ of a given aircraft, then the total additional cost $C_{\mathcal{P}}$ is then calculated using the Equation (11).

$$
C_{\mathcal{P}}=\sum_{f l t \in \mathcal{P}} C_{f l t}
$$

In order the represent as precisely as possible an actual flight program, we focus the study on the reference airplane and use the flight corresponding program observed using the enriched MOZAIC database. Both the airplane and the database are presented in Section III] In order to reduce the calculation time, the flight program is reduced to a sample of 1000 flights randomly chosen among the MOZAIC flights and noted $\mathcal{P}_{1000}$. For a long range airplane like the reference airplane, this roughly corresponds to three years of operations.

\section{MDO process with take-off requirements as design variables}

Moving the take-off requirements from constraints to design variables in the optimization problem necessitate to modify the objective function to take into account the additional cost arising the loss of performances due to relaxing these requirements. Indeed, without these modifications, the optimization algorithm would only measure the benefit of removing a constraint and would only increase the TOFL and reduce the altp and the $\triangle T_{I S A}$, which would have the tendency to allow to reduce even more the wing surface and the engine thrust. This is not acceptable as the future airplane will then loose the capability to take-off from a greater number of airport. This may prevent the airlines to provide the transportation service on a large part of their network. This effect must therefore be measured thanks to the model developed and presented in Section IV.C and integrated in the modified design process.

The modified design process we propose includes both benefits and additional costs resulting from less stringent take-off requirements is presented in Figure 14. Based on the results obtained in Section IV.B. we modify the MDO process presented in Section IV.A. We recall here the Equation (9) in order to emphasize the evolution. 


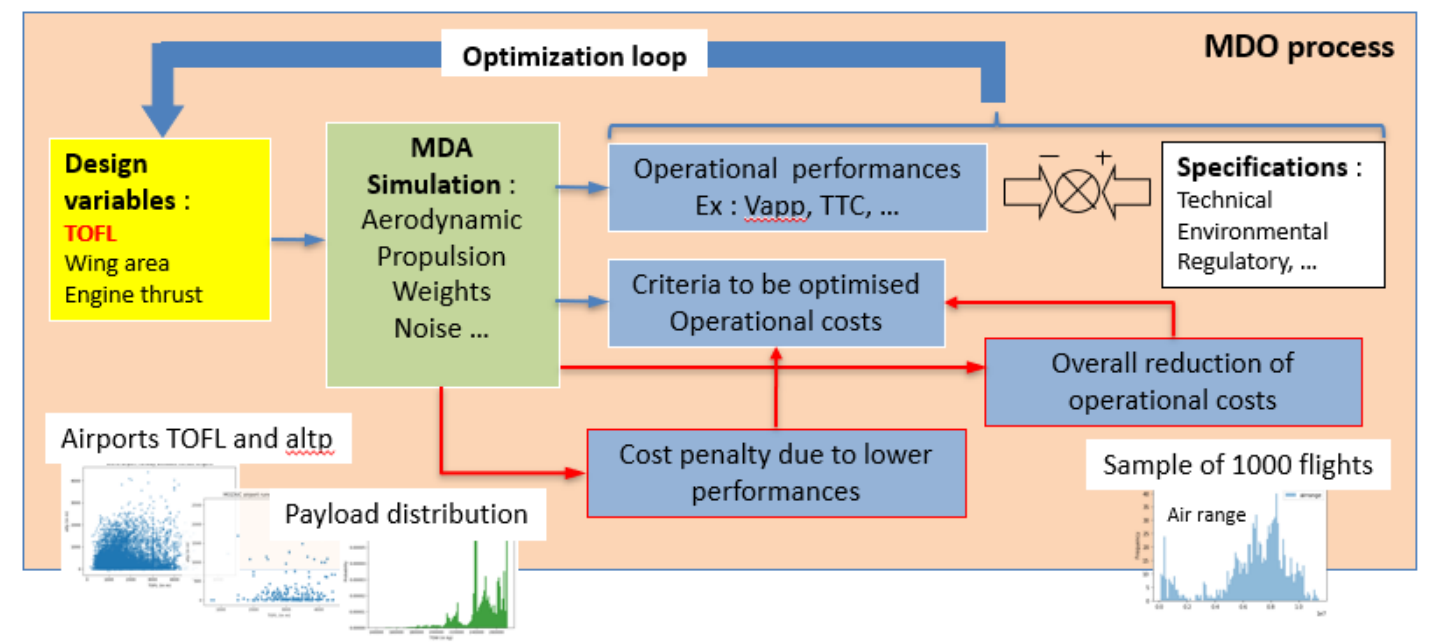

Fig. 14 Modified design process with take-off requirements turned into design variables.

$$
\begin{array}{ll}
\min _{x} & f(x) \\
\text { s.t. } & g(x) \geq 0
\end{array}
$$

where:

- $x$, the design variable vector, includes now the wing reference area wing_area the engine sea level static thrust $(S L S T)$ and the take-off field length $T O F L$ at an altitude altp $=0 \mathrm{ft}$ and a temperature $\triangle T_{I S A}=0 \operatorname{deg} C$. These conditions are referred to as the sea level standard (sls) take-off conditions.

- $f(x)$, the objective function combines the cash operating cost $C O C$ and the additional cost presented in Section IV.C

- $g(x)$, the inequality constraint vector, does not include anymore the take-off field length (TOFL) and reduces to the set composed with the approach speed (App_speed), the one-engine-inoperative path after take-off (OEI_path), the climb and cruise ceiling vertical speeds $\left(V z_{-} M C L\right.$ and $\left.V z_{-} M C R\right)$ and the time-to-climb to cruise altitude $(T T C)$. The values given to these constraints remain unchanged.

The objective function must provide an answer to the following question: will the new designed airplane cost less to the airlines as the reference one? It then combines both the total cash operating cost and the total additional costs arising from the potentially reduced take-off performances. It is represented by the Equation $(12)$.

$$
f(x)=\sum_{f l t \in \mathcal{P}_{1000}}\left(\operatorname{COC}_{f l t}+C_{f l t}\right)
$$

Some important hypothesis have been made to calculate the objective function:

- The flight program remains exactly identical to the one observed for the reference aircraft.

- The exact same payloads as those estimated in Section III.B.2 have to be transported on each flight.

- The meteorological conditions remain unchanged.

- Any other operational uncertainty affecting for example the effective air range is unchanged.

- The flight conditions such as the mach and the cruise altitude remain the same.

\section{E. Review of the design space}

The design space available to find a new optimal solution is restricted. As we consider a reference aircraft and its operations, the objective is to find a better airplane that would reduce the airline operating costs represented by the objective function in Equation (12). As shown in Figure 6, the reference aircraft is the solution of an optimization process. This situation is equivalent to the Figure $15 \mathrm{~b}$ The TOFL is equal to 3259 meters in $s l s$ conditions. With these take-off performances, the reference aircraft can carry the intended payload with no restriction. On one side, if we 
consider any shorter take-off runway (as shown in Figure 15c), then the take-off performances will be improved. The airplane will then be able to take-off with a higher payload but, as a result of the hypothesis made, no additional payload is ready to embark. The airplane is also heavier and the $C O C$ is increased although no additional revenue compensate it. On Figure 15b, the improved design is not on the right side of the reference aircraft optimum. On the other side, if we consider longer take-off runway (as shown in Figure 15a), the take-off performance reduces. The airplane is lighter with less powerful engines which enable the airlines to save money on the $C O C$ for all the flights of the program $\mathcal{P}_{1000}$. Nevertheless, an increasing amount of payload cannot be loaded on-board and is left behind, which generates extra costs for the airline. Figure 16 illustrates this situation. Furthermore, if the TOFL is increased too much, it has no effect anymore on the design. Indeed, the new airplane has to satisfy other performance constraints that will become active. Figure 15 a shows the extreme case where the TOFL reaches the limit of its influence on the design.

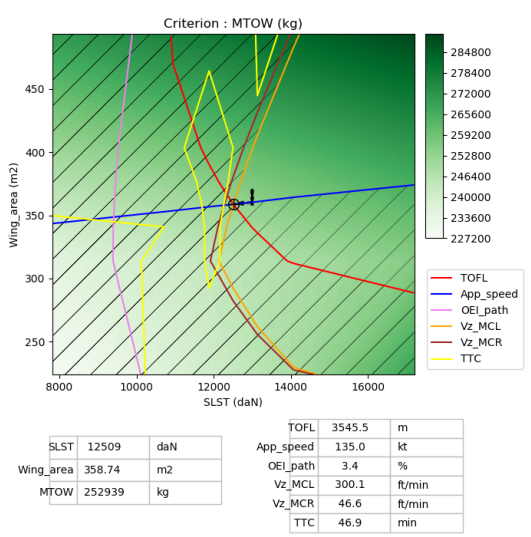

(a) Design space for $T O F L=3545 m$

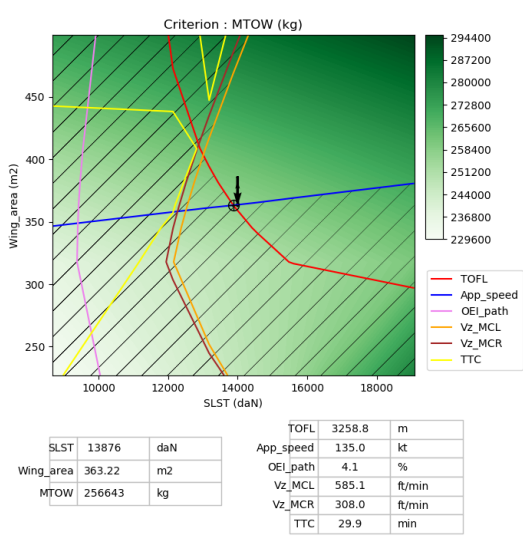

(b) Design space for $T O F L=3259 m$

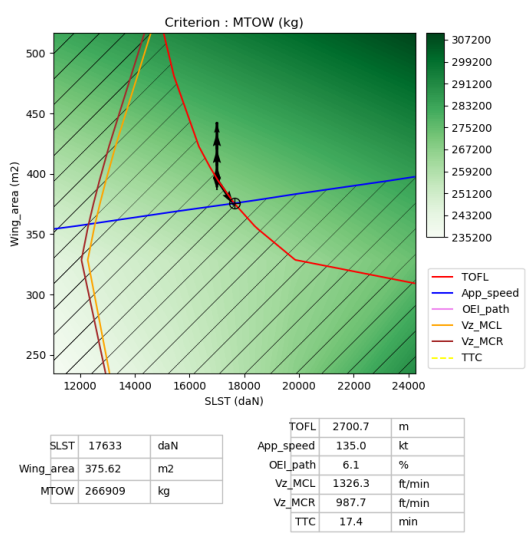

(c) Design space for $T O F L=2700 \mathrm{~m}$

Fig. 15 Design space and optimum aircraft for three take-off requirements (in sls conditions: altp $=0 \mathrm{ft}$ and $\Delta T_{I S A}=0 \operatorname{deg} C$ ).
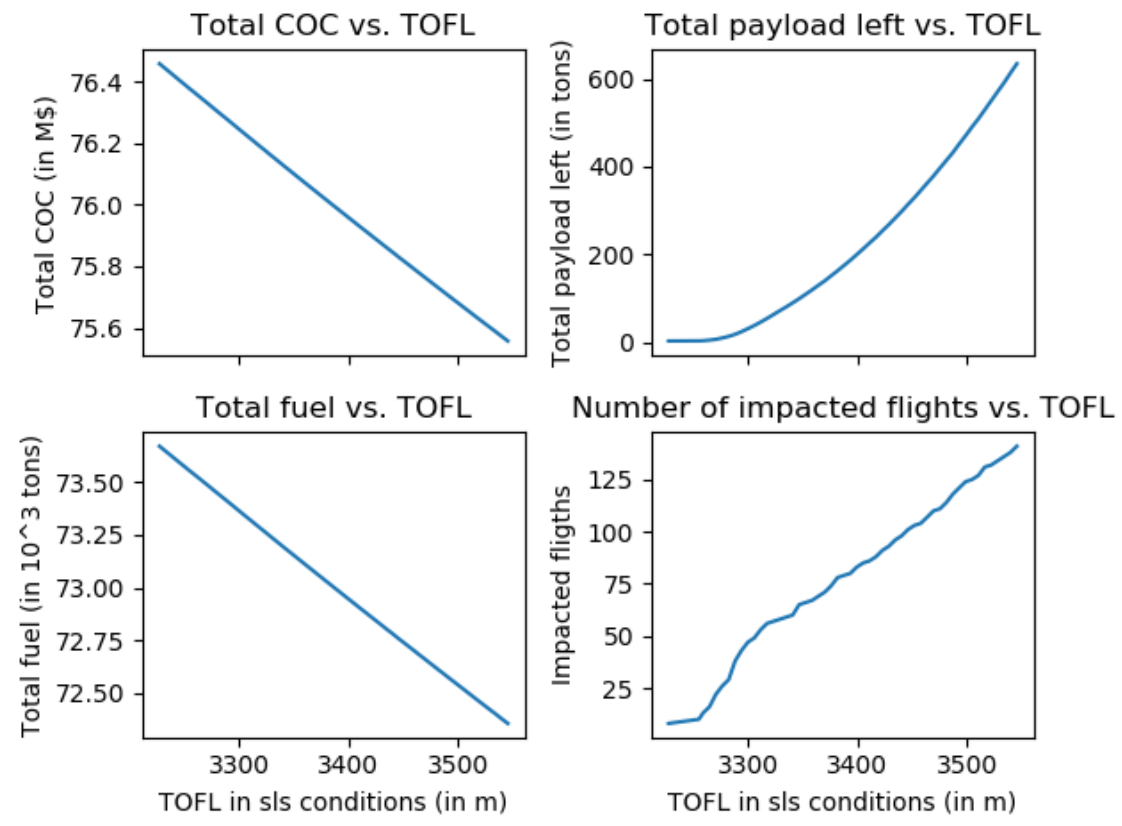

Fig. 16 Evolution of the $C O C$, the payload left behind, the total fuel and the number of impacted flights versus the TOFL in $s l s$ conditions. 


\section{F. Results of the optimization}

Tables 7 and 8 present the results of the optimization process. The first table shows the values found and the second shows the relative difference with the reference airplane. The values are presented for various values of the parameter $c_{p l d}$ that represents the additional per kilogram of payload that could not be loaded on-board.

\begin{tabular}{|c|c|c|c|c|}
\hline$c_{p l d}$ (in $\left.\$ / \mathrm{kg}\right)$ & TOFL (in m) & wing_area $\left(\right.$ in $\left.\mathrm{m}^{2}\right)$ & SLST (in N) & Total cost (in M\$) \\
\hline 0.0 & 3535.50 & 358.9 & 125525 & 75.5843 \\
0.5 & 3535.50 & 358.9 & 125525 & 75.8828 \\
1.0 & 3452.15 & 360.1 & 129239 & 76.1425 \\
1.5 & 3370.33 & 361.4 & 133104 & 76.2534 \\
2.0 & 3316.05 & 362.2 & 135794 & 76.3045 \\
2.5 & 3298.57 & 362.5 & 136683 & 76.3246 \\
3.0 & 3289.20 & 362.7 & 137174 & 76.3363 \\
4.0 & 3283.18 & 362.8 & 137479 & 76.3534 \\
5.0 & 3277.43 & 362.9 & 137786 & 76.3666 \\
6.0 & 3272.29 & 363.0 & 138049 & 76.3760 \\
8.0 & 3269.26 & 363.0 & 138205 & 76.3912 \\
10.0 & 3266.76 & 363.1 & 138339 & 76.4045 \\
\hline
\end{tabular}

Table 7 Results of the optimization

\begin{tabular}{|c|c|c|c|c|}
\hline$c_{p l d}$ (in $\left.\$ / \mathrm{kg}\right)$ & TOFL & wing_area & SLST & Total cost \\
\hline 0.0 & $8.48 \%$ & $-1.19 \%$ & $-9.53 \%$ & $-1.02 \%$ \\
0.5 & $8.48 \%$ & $-1.19 \%$ & $-9.53 \%$ & $-0.63 \%$ \\
1.0 & $5.93 \%$ & $-0.86 \%$ & $-6.85 \%$ & $-0.30 \%$ \\
1.5 & $3.42 \%$ & $-0.51 \%$ & $-4.07 \%$ & $-0.15 \%$ \\
2.0 & $1.75 \%$ & $-0.27 \%$ & $-2.13 \%$ & $-0.09 \%$ \\
2.5 & $1.21 \%$ & $-0.19 \%$ & $-1.49 \%$ & $-0.07 \%$ \\
3.0 & $0.93 \%$ & $-0.14 \%$ & $-1.14 \%$ & $-0.05 \%$ \\
4.0 & $0.74 \%$ & $-0.11 \%$ & $-0.92 \%$ & $-0.04 \%$ \\
5.0 & $0.57 \%$ & $-0.09 \%$ & $-0.69 \%$ & $-0.03 \%$ \\
6.0 & $0.41 \%$ & $-0.06 \%$ & $-0.51 \%$ & $-0.02 \%$ \\
8.0 & $0.31 \%$ & $-0.05 \%$ & $-0.39 \%$ & $-0.01 \%$ \\
10.0 & $0.24 \%$ & $-0.04 \%$ & $-0.30 \%$ & $-0.01 \%$ \\
\hline
\end{tabular}

Table 8 Results of the optimization presented as relative difference compared to the reference aircraft.

We can see that if this value is very small, it is more interesting to alleviate the take-off requirements. The TOFL can be increased up to $8.48 \%$. In this case, the gain in COC can be improved by $0.63 \%$. This may look marginal but when we scale that result to a full fleet on a long period of time, it can represent interesting gains. However, we also see that as soon as the $c_{p l d}$ increases, the gains reduce fast. This can certainly be explained by the fact that the airlines work hard to maximize the use of their airplanes, once they are aware of their operational limits. The values we explore for the $c_{\text {pld }}$ parameter range from $0 \$ / \mathrm{kg}$ up to $10 \$ / \mathrm{kg}$. If we consider a passenger represents $100 \mathrm{~kg}$ of payload, then we cover a range of penalty up to $1000 \$$ per passenger which appears reasonable compared to the price of a flight ticket for a long range flight. It is important to note that these costs will greatly depend on the region of the world, the airlines, the period of the year, the day of the week and the services related to the ticket. When increasing the TOFL, the wing area and the $S L S T$ both reduce. The $S L S T$ reduces more rapidly and can get to few percent reductions. The impact on the price of the airplane and the engine is not part of this work but we can expect that these reductions would make the 
airplane more affordable.

\section{Conclusion}

The take-off requirements often appear as very constraining from an airplane design point of view. The objective of the work presented was to challenge these requirements. An original and significant effort has been made to make this analysis as close as possible to the actual operational world, by considering and modeling a real aircraft and observing its operations in details. Both the calibration of the overall airplane conceptual design tool MARILib to make it specific to a given airplane and the enrichment of the MOZAIC database where the mass was an essential missing parameter were the opportunity to test machine learning methods like the random forest approach. The take-off requirements are not limited to the take-off field length but include other parameters. The altitude of the airport, the temperature, the wind and the runway condition (wet or dry) are important parameters. Our analysis focused on the take-off field length, the altitude and the temperature. In order to identify the most important one in terms of impact on the operational costs, sensitivity analysis was carried using the Sobol indices as well as new indices based on Cramér von Mises distance and that take into account the full distribution of the output rather than only its variance. The take-off field length was found to be the most important take-off parameter. The usual multidisciplinary optimization where the take-off requirements appear as a constraint was modified. The take-off field length was then included in the design variable vector and the objective function was modified to include a cost penalty due to reduced operational performances, otherwise there would have been not balancing to the increase of the take-off field length. The multidisciplinary analysis was also adjusted to estimate this cost penalty using data-driven models. The enriched operational data played an important role as the estimation greatly relied on them. Finally, the results of the optimization show that the gains represent less than $0.5 \%$ of the the total cash operating costs. Additional costs reduction could have been considered if the modification of the airline flight program and a new optimization of the flight profile had been included in the analysis. From a methodological stand point, the work presented in this paper shows it is possible to tie the link between the airplane design processes and the operational world, by developing data-driven models. The use of new sensitivity indices also appeared as a key point to better estimate the important operational features to be considered in the design process.

\section{Acknowledgments}

This work is part of the activities of ONERA - ISAE - ENAC joint research group and was made in cooperation with the Hong-Kong University of Science and Technology under the travel grant project No42802XB (France) and F-HKUST602/18 (Hong Kong) funded by the PROCORE-France/Hong Kong Joint Research Scheme 2018/19.

We want to thank Prof. Christian Bes from the University of Toulouse and Prof. Marcel Mongeau from the ENAC, Toulouse, for their support when elaborating this work. We also want to thank François Gallard from the IRT Saint-Exupéry, Toulouse, for his help in elaborating the calibration process.

MOZAIC/CARIBIC/IAGOS data were created with support from the European Commission, national agencies in Germany (BMBF), France (MESR), and the UK (NERC), and the IAGOS member institutions (http://www.iagos.org/partners). The participating airlines (Lufthansa, Air France, Austrian, China Airlines, Iberia, Cathay Pacific, Air Namibia, Sabena) supported IAGOS by carrying the measurement equipment free of charge since 1994 . The data are available at http://www.iagos.fr thanks to additional support from AERIS.

\section{References}

[1] Druot, T. Y., Belleville, M., Roches, P., Gallard, F., Peteilh, N., and Gazaix, A., "A Multidisciplinary Airplane Research Integrated Library With Applications To Partial Turboelectric Propulsion,” AIAA Aviation 2019 Forum, 2019, p. 3243.

[2] European Research Infrastructure IAGOS (In-service Aircraft of a Global Observing System), "MOZAIC IAGOS Database,”, 2016. URL http://www.iagos.org/

[3] Peteilh, N., Mongeau, M., Bes, C., Druot, T., and Conderolle-Lestremau, M., Modeling Operational Variability for Robust Multidisciplinay Design Optimization, American Institute of Aeronautics and Astronautics, 2017. doi:doi:10.2514/6.2017-4328, URL https://doi.org/10.2514/6.2017-4328

[4] Hastie, T., Tibshirani, R., and Friedman, J., The elements of statistical learning: data mining, inference, and prediction, Springer Science \& Business Media, 2009. 
[5] Breiman, L., Classification and regression trees, Routledge, 2017.

[6] Sobol, I. M., "Sensitivity estimates for nonlinear mathematical models," Mathematical modelling and computational experiments, Vol. 1, No. 4, 1993, pp. 407-414.

[7] Gamboa, F., Janon, A., Klein, T., Lagnoux, A., et al., "Sensitivity analysis for multidimensional and functional outputs," Electronic Journal of Statistics, Vol. 8, No. 1, 2014, pp. 575-603.

[8] Gamboa, F., Klein, T., and Lagnoux, A., "Sensitivity analysis based on Cramér von Mises distance," SIAM/ASA Journal on Uncertainty Quantification, Vol. 6, No. 2, 2018, pp. 522-548. URL https://hal.archives-ouvertes.fr/hal01163393\% CNRS\% INSMI\% IMT\% UNIV-TLSE2\% INSA-TOULOUSE\% ENAC\% DEVI\% INSA-GROUPE\% UNIV-TLSE3

[9] Blatman, G., and Sudret, B., "Efficient computation of global sensitivity indices using sparse polynomial chaos expansions," Reliability Engineering \& System Safety, Vol. 95, No. 11, 2010, pp. 1216-1229.

[10] Janon, A., Klein, T., Lagnoux, A., Nodet, M., and Prieur, C., "Asymptotic normality and efficiency of two sobol index estimators,” ESAIM: Probability and Statistics, Vol. 18, 2014, pp. 342-364. 